\title{
Classifications of Computable Structures
}

\author{
Karen Lange and Russell Miller \\ and Rebecca M. Steiner
}

\begin{abstract}
Let $\mathcal{K}$ be a family of structures, closed under isomorphism, in a fixed computable language. We consider effective lists of structures from $\mathcal{K}$ such that every structure in $\mathcal{K}$ is isomorphic to exactly one structure on the list. Such a list is called a computable classification of $\mathcal{K}$, up to isomorphism. Using the technique of Friedberg enumeration, we show that there is a computable classification of the family of computable algebraic fields, and that with a $\mathbf{0}^{\prime}$-oracle, we can obtain similar classifications of the families of computable equivalence structures and of computable finite-branching trees. However, there is no computable classification of the latter, nor of the family of computable torsion-free abelian groups of rank 1, even though these families are both closely allied with computable algebraic fields.
\end{abstract}

\section{Introduction}

Classification of structures up to isomorphism is a common goal in all areas of mathematics. Here, following work of Goncharov and Knight in [10], we examine classification questions from the perspective of computable structure theory. Specifically, we are interested in effective classifications of fixed families of structures. Throughout, we examine families $\mathcal{K}$ of computable structures, closed under isomorphism, in a fixed language. (Recall that a structure $\mathcal{A}$ is computable if the atomic diagram of $\mathcal{A}$, denoted $\mathcal{D}(\mathcal{A})$, is computable. See [1] for general background on computable structure theory.) It is natural to ask which such families of structures have effective classifications.

Definition 1.1 Let $\mathcal{K}$ be a family of computable structures, closed under isomorphism, in a fixed computable language.

A computable enumeration of $\mathcal{K}$ consists of a computable function $f$ such that, for every $n \in \omega, f(n)$ is a computable index of some structure in $\mathcal{K}$ (i.e.

2010 Mathematics Subject Classification: Primary 03C57, 03D45

Keywords: Friedberg enumeration, computable structure theory, equivalence structure, effective classification 
$\varphi_{f(n)}=\chi_{D(\mathcal{A})}$ for some $\left.\mathcal{A} \in \mathcal{K}\right)$, and for every $\mathcal{A} \in \mathcal{K}$, there is some $n \in \omega$ such that $f(n)$ is a computable index for a structure $\mathcal{M}$ isomorphic to $\mathcal{A}$ (i.e. $\varphi_{f(n)}=\chi_{D(\mathcal{M})}$ with $\left.\mathcal{M} \cong \mathcal{A}\right)$.

A computable classification of $\mathcal{K}$ is a computable enumeration of $\mathcal{K}$ such that each structure in $\mathcal{K}$ is isomorphic to exactly one structure in the enumeration.

(Our notation for partial computable functions $\varphi_{e}$ and other computability concepts follows [18].) Many strongly minimal theories provide examples of families $\mathcal{K}$ with computable classifications.

Example 1.2 Each of the following families has a computable classification.

1. Computable algebraically closed fields (either in a fixed characteristic or over all characteristics).

2. Computable vector spaces over a fixed computable field.

3. Computable successor structures, i.e. models of $T h(\mathbb{Z}, S)$ where $S$ is the successor function on $\mathbb{Z}$.

Goncharov and Knight [10] asked whether computable classifications exist for other families of structures, and in particular for the family of computable equivalence structures. (Among families not defined by strongly minimal theories, this family is widely considered to be one of the simplest possible examples.) Although this question remains open, they answered it for a subfamily of these structures, in [10, Theorem 5.5].

Theorem 1.3 (Goncharov \& Knight [10]) There is a computable classification of the family of computable equivalence structures with infinitely many infinite equivalence classes.

Computable classification problems have natural connections to index set and isomorphism problems for families of computable structures. We refer the reader to [1] for background on index sets and isomorphism problems and to [4] for examples.

Definition 1.4 Let $\mathcal{K}$ be a family of computable structures, closed under isomorphism, in a fixed language.

(i) We define the index set of $\mathcal{K}$ to be the set $I(\mathcal{K})$ of computable indices for models in $\mathcal{K}$, i.e.

$$
I(\mathcal{K})=\left\{e \in \omega \mid(\exists \mathcal{A} \in \mathcal{K})\left[\varphi_{e}=\chi_{D(\mathcal{A})}\right]\right\} .
$$

If $i \in I(\mathcal{K})$, let $\mathcal{A}_{i}$ be the (presentation of the) structure in $\mathcal{K}$ such that $\varphi_{i}=\chi_{D\left(\mathcal{A}_{i}\right)}$.

(ii) We call the set

$$
\left\{\langle i, j\rangle \in \omega \mid i, j \in I(\mathcal{K}) \& \mathcal{A}_{i} \cong \mathcal{A}_{j}\right\}
$$

the isomorphism problem of $\mathcal{K}$.

In [10, Prop. 5.8], Goncharov and Knight observed the first statement below which places a restriction on the existence of computable classifications. We prove a strong version of the second statement in Corollary 3.3.

\section{Proposition 1.5}

1. (Goncharov \& Knight [10]) No family $\mathcal{K}$ whose isomorphism problem is $\Sigma_{1}^{1}$-complete and whose index set is hyperarithmetic has a computable classification, nor even a hyperarithmetic classification. 
2. For each $n \in \omega$, no family $\mathcal{K}$ whose whose index set is $\Delta_{n}^{0}$ and whose isomorphism problem is $\Sigma_{n}^{0}$-complete has a computable classification.

Part 1 of Proposition 1.5 yields many examples of families lacking computable classifications.

Example 1.6 The following families of computable structures do not have computable classifications.

1. Graphs

2. Fields

3. Any of the families described by Hirschfeldt, Khoussainov, Shore, and Slinko in [12], including computable partial orders, lattices, rings, groups, and integral domains.

In Corollary 3.3, we use an extension of Part 2 of Proposition 1.5 to show that there is no computable classification of computable torsion-free abelian groups of rank 1. In contrast, for families $\mathcal{K}$ whose isomorphism problem is $\Pi_{n}^{0}$-complete (for some $n \in \omega$ ), the only way we have found to prove that $\mathcal{K}$ has no computable classification is to show that it does not even have a computable enumeration. This method is used in $\S 4$ and $\S 5.1$.

1.1 Our methods. Friedberg's 1958 proof of the existence of a computable enumeration of all c.e. sets without repetition [8] provides our main technique for establishing positive results on classifications. From the perspective of computable structure theory, Friedberg's result can be thought of as finding a computable classification of the $\Sigma_{1}^{0}$-definable subsets of $\mathbb{N}$, up to equality. His technique is important in the study of numberings or enumerations, which has taken place mainly in the former Soviet Union; see for example [7]. Other researchers have studied whether such enumerations exist for other families of sets of a given complexity (see for example [11]).

We will apply Friedberg's approach to prove the existence of certain effective classifications. In this paper, we focus on four families of computable structures: algebraic fields, torsion-free abelian groups of rank 1, finite-branching trees, and equivalence structures. Algebraic fields prove to be tractable using the Friedberg method, and this leads us to consider the related families of abelian groups and trees. We find Friedberg's technique useful for the trees as well, and for computable equivalence structures, but to apply it there, we need more computational power. Therefore, we relativize Definition 1.1 to other Turing degrees, in order to develop a fuller understanding of effective classifications for these families.

Definition 1.7 Let $\boldsymbol{d}$ be a Turing degree and $C$ a subset of $\omega$. We say $e \in \omega$ is a $C$-computable index for a structure $\mathcal{A}$ if $\Phi_{e}^{C}=\chi_{D(\mathcal{A})}$. A $\boldsymbol{d}$-computable classification of $\mathcal{K}$ by $C$-computable indices is a uniformly $\boldsymbol{d}$-computable enumeration of $C$-computable indices for structures in $\mathcal{K}$ such that each structure in $\mathcal{K}$ is represented exactly once in the enumeration up to isomorphism.

In all cases we will consider in this article, either $C=\emptyset$ or $C \in \boldsymbol{d}$. That is, we study $\boldsymbol{d}$-computable classifications either by computable indices or by $\boldsymbol{d}$-computable indices. For us, $\boldsymbol{d}$ will always be of the form $\mathbf{0}^{(n)}$. Notice that in the definition, when $C \equiv_{T} \widetilde{C}$, a number $e$ may be a $C$-computable index for $\mathcal{A}$ without being a $\widetilde{C}$-computable index for $\mathcal{A}$. However, in this situation, 
there are computable total injective functions $f$ and $\tilde{f}$ such that, for every such $e$ and $\tilde{e}, \Phi_{e}^{C}=\Phi_{f(e)}^{\widetilde{C}}$ and $\Phi_{\tilde{e}}^{\widetilde{C}}=\Phi_{\tilde{f}(\tilde{e})}^{C}$. Hence it is reasonable to speak of $\boldsymbol{d}$-computable indices without specifying the oracle set in $\boldsymbol{d}$.

1.2 Families to be studied. We consider algebraic fields in $\S 2$, torsion-free abelian groups in $\S 3$, finite-branching trees in $\S 4$, and equivalence structures in $\S 5$.

1.2.1 Algebraic fields. Since the isomorphism problem for computable fields of characteristic 0 is $\Sigma_{1}^{1}$-complete and the index set of such fields is only $\Pi_{2}^{0}$, Proposition 1.5 gives the following result.

Proposition 1.8 There is no hyperarithmetic classification of all computable fields.

However, when we restrict ourselves to algebraic computable fields, we fare much better. Recall that a field is algebraic if every element of the field satisfies a nonzero polynomial over the prime subfield (which is either $\mathbb{Q}$ or $\mathbb{F}_{p}$, depending on the characteristic of the field). In $\S 2.2$, we will use Friedberg's method to prove the following theorem.

Theorem 1.9 There is a computable classification of the family of computable algebraic fields.

1.2.2 Torsion-free abelian groups of rank 1. We write $\mathbf{T F A} \mathbf{b}_{1}$ for the family of computable torsion-free abelian groups of rank 1 . These are precisely the c.e. subgroups of a computable presentation of the group $(\mathbb{Q},+)$, which allows us to enumerate them computably. However, we show that there is no computable classification of $\mathbf{T F A} \mathbf{b}_{1}$. Indeed, we prove that $\mathbf{0}^{(n)}$-computable classifications of $\mathbf{T F A} \mathbf{b}_{1}$ by computable indices exist only for $n \geq 3$.

Theorem 1.10 There is no $\mathbf{0}^{\prime \prime}$-computable classification of $\boldsymbol{T F A \boldsymbol { b } _ { 1 }}$ by computable indices, but there does exist a $\mathbf{0}^{\prime \prime \prime}$-computable classification of $\mathbf{T F A \boldsymbol { b } _ { 1 }}$ by computable indices.

We prove the existence portion of Theorem 1.10 in Lemma 3.4 and the nonexistence portion in Corollary 3.3.

1.2.3 Finite-branching trees. For our purposes, a tree $T$ is a substructure of the structure $\omega^{<\omega}$ of all finite strings of natural numbers. The language contains just a unary function, the predecessor function $P$, which names the immediate predecessor of each element (and maps the root to itself). Note that $P$ is computable on $\omega^{<\omega}$. To be a tree, $T$ must be nonempty and closed under $P$, and to be computable, $T$ must be a computable subset of $\omega^{<\omega}$. (Our discussion does not necessarily carry over to computable trees in the language of partial orders.) Finally, $T$ is finite-branching if, for each $x \in T$, the preimage of $x$ under $P$ is finite. We use $\mathcal{T}$ to denote the family of all computable finite-branching trees.

Finite-branching trees are algebraic, in the model-theoretic sense of the word, and have been shown in [19] to have properties very similar to those of algebraic fields. However, we will show that there is no computable enumeration of $\mathcal{T}$, let alone any computable classification of this family. (In fact, we show more; see Proposition 4.2). On the other hand, in Proposition 4.4 we will use Friedberg's method to give a classification of $\mathcal{T}$ using a $\mathbf{0}^{\prime}$-oracle, 
in a way which is not known to be possible for $\mathbf{T F A} \mathbf{b}_{1}$. The theorem below follows from Propositions 4.2, 4.3, and 4.4.

Theorem 1.11 There exists a $\mathbf{0}^{\prime \prime \prime}$-computable classification of $\mathcal{T}$ by computable indices as well as a $\mathbf{0}^{\prime}$-computable classification of $\mathcal{T}$ by $\mathbf{0}^{\prime}$-computable indices. However, no classification of $\mathcal{T}$ by computable indices can be $\mathbf{0}^{\prime \prime}$ computable.

1.2.4 Equivalence structures. Goncharov and Knight examined computable equivalence structures in [10], as noted above. (A countable equivalence structure is simply an equivalence relation on the domain $\omega$.) They gave a computable classification of the family $\mathcal{E}_{\infty}$ of all computable equivalence structures that contain infinitely many infinite equivalence classes. The same problem for the family $\mathcal{E}_{n}$ of computable equivalence structures with exactly $n$ infinite classes proves thornier, and we show in $\S 5.1$ that there is no computable enumeration (let alone classification) of any family $\mathcal{E}_{n}$. However, in $\S 5.2$, we produce a $\mathbf{0}^{\prime}$-computable classification of $\mathcal{E}_{0}$ using $\mathbf{0}^{\prime}$-computable indices, applying Friedberg's method once again, relativized to a $\mathbf{0}^{\prime}$-oracle and starting with a particular $\mathbf{0}^{\prime}$-computable enumeration of $\mathcal{E}_{0}$. From this result, we readily produce a $\mathbf{0}^{\prime}$-computable classification of the entire family $\mathcal{E}$ of all computable equivalence relations, again using $\mathbf{0}^{\prime}$-computable indices. The oracle $\mathbf{0}^{\prime}$ is not particularly powerful so we regard this result as a vindication of the view that computable equivalence structures, while nontrivial, are not a particularly complex family of structures.

\section{Fields by Friedberg}

After discussing some necessary background in $\S 2.1$, we give a computable classification of the family of algebraic fields in $\S 2.2$, proving Theorem 1.9.

2.1 Background on fields Recall that the splitting set $S_{F}$ of a computable field $F$ is the set of reducible polynomials in $F[X]$. (Formally, it is the set of code numbers for these polynomials when $F[X]$ is listed out in the canonical way from the computable presentation of $F$.) The Turing degree of the splitting set does not vary between computable presentations of a single algebraic field, and $S_{F}$ is Turing-equivalent to the root set $R_{F}$, the set of those polynomials in $F[X]$ having roots in $F$. If $S_{F}$ is computable, then $F$ is said to have a splitting algorithm, and this algorithm allows one to identify the irreducible polynomials in $F[X]$. Finally, there is a computable presentation of the algebraic closure $\bar{F}$ of $F$ : this presentation may be given uniformly in an index for $F$, as may an index for a computable embedding $g: F \rightarrow \bar{F}$, and the image $g(F)$ of $F$ within $\bar{F}$ is Turing-equivalent to $S_{F}$. Hence $g(F)$ is computable if and only if $F$ has a splitting algorithm. All of this follows essentially from Rabin's Theorem (see [17]).

We take advantage of the following facts.

Lemma 2.1 For each characteristic $p \geq 0$, there is a computable enumeration $\left\langle F_{e}\right\rangle_{e \in \omega}$ of all computable algebraic fields of characteristic $p$.

Proof. Fix a computable presentation $\bar{Q}$ of the algebraic closure of the prime field $Q\left(=\mathbb{Q}\right.$ or $\left.=\mathbb{F}_{p}\right)$ of characteristic $p$. For each $e$, let $F_{e}$ be the subfield of $\bar{Q}$ generated by the c.e. set $W_{e}$. Thus, each $F_{e}$ is itself c.e., uniformly 
in $e$, and the fields $F_{e}$ form a computable enumeration of all computably presentable algebraic fields of characteristic $p$ (since every such field has a computable embedding into $\bar{Q}$, with c.e. image). Notice that, while $F_{e}$ itself may not be technically a computable field (if its domain, which is c.e., fails to be computable), it is computably isomorphic to a computable field, just by taking a 1-1 computable enumeration of its elements and pulling back the field operations to the domain of this enumeration. Of course, in positive characteristic, we allow finite computable fields in our enumeration.

The following lemma appears as [16, Corollary 3.9], and also (with a different proof) in [9, Appendix A]. Essentially it follows from König's Lemma.

Lemma 2.2 Two algebraic fields $E$ and $F$ of characteristic 0 are isomorphic if and only if, for all finitely generated algebraic field extensions $K$ of $\mathbb{Q}$, the field $K$ embeds in $E$ if and only if $K$ embeds in $F$.

2.2 Friedberg's Construction We now recast Friedberg's construction of a classification of all c.e. sets in terms of classifying some family of $\boldsymbol{d}$-computably presentable structures of a given kind. We then apply this construction to computable algebraic fields.

Given a structure $\mathcal{M}$ with domain $\subseteq \omega$, we let $\mathcal{M} \uparrow s$ be the substructure of $\mathcal{M}$ generated by the elements $\{0,1, \ldots, s-1\} \cap \operatorname{dom}(\mathcal{M})$ under the function symbols in the language. Since we allow function symbols, $\mathcal{M} \uparrow s$ need not be finite. In general its domain may only be computably enumerable, but we treat it as an $\mathcal{M}$-computable structure, since we get an $\mathcal{M}$-computable isomorphism from each $\mathcal{M} \uparrow s$ onto a computable structure, uniformly in $s$, by mapping an initial segment of $\omega$ onto the domain of $\mathcal{M} \uparrow s$. We do specifically allow $\mathcal{M}$ to have finite domain; this is important when dealing with fields in positive characteristic, and also for equivalence structures in $\S 5.2$. We also say $\mathcal{M}_{i} \uparrow s$ is a proper substructure of $\mathcal{M}_{j} \backslash t$ if the former embeds into the latter but they are not isomorphic. (An embedding is just an injective homomorphism.)

Theorem 2.3 Let $\boldsymbol{d}$ be a Turing degree, and $\mathcal{K}$ a family of structures, closed under isomorphism, in a fixed $\boldsymbol{d}$-computable language. Suppose there exists a $\boldsymbol{d}$-computable enumeration $\left\langle\mathcal{M}_{i}\right\rangle_{i \in \omega}$ of $\mathcal{K}$ by $\boldsymbol{d}$-computable indices satisfying the following conditions.

1. For each $\mathcal{M}_{i}$ and each stage $s$,

(a) $\mathcal{M}_{i} \uparrow s$ is an element of $\mathcal{K}$ and

(b) there is some $t>s$ and $j \in \omega$ such that

- $\mathcal{M}_{i}\left\lceil s\right.$ is a proper substructure of $\mathcal{M}_{j}\lceil t$ and

- for all $\left.k<s, \mathcal{M}_{j}\right\rceil t$ is not isomorphic to $\mathcal{M}_{k}\lceil s$.

2. (a) For every two indices $i$ and $j, \mathcal{M}_{i} \cong \mathcal{M}_{j}$ iff $i$ and $j$ satisfy:

$(\forall s)(\exists t)\left[\mathcal{M}_{i}\left\lceil s\right.\right.$ embeds into $\mathcal{M}_{j}\left\lceil t \quad \& \quad \mathcal{M}_{j}\left\lceil s\right.\right.$ embeds into $\mathcal{M}_{i}\lceil t]$.

(b) The following two sets are both $\boldsymbol{d}$-computable.

$$
\begin{aligned}
& \left\{\langle i, t, j, s\rangle: \mathcal{M}_{i} \uparrow t \cong \mathcal{M}_{j}\lceil s\}\right. \\
& \left\{\langle i, t, j, s\rangle: \mathcal{M}_{i} \uparrow t \text { embeds into } \mathcal{M}_{j} \uparrow s\right\}
\end{aligned}
$$

Thus, the isomorphism problem and the proper substructure problem for any two structures $\left.\mathcal{M}_{i}\right\rceil t$ and $\mathcal{M}_{j}\lceil s$ are $\boldsymbol{d}$-computable. 
Then there is a $\boldsymbol{d}$-computable classification by $\boldsymbol{d}$-computable indices of the structures in $\mathcal{K}$.

Proof. Let $\left\langle\mathcal{M}_{i}\right\rangle_{i \in \omega}$ be a $\boldsymbol{d}$-computable enumeration of all structures in a family $\mathcal{K}$ by $\boldsymbol{d}$-computable indices satisfying the assumptions listed in the theorem. We construct a $\boldsymbol{d}$-computable classification $\left\langle\mathcal{N}_{i}\right\rangle_{i \in \omega}$ by $\boldsymbol{d}$-computable indices of the structures in $\mathcal{K}$ by employing Friedberg's method. For the reader's convenience, we imitate Friedberg's original construction in [8, Thm. 3] as closely as possible. In particular, at times we will assign $\mathcal{N}_{k}$ to be a follower of some $\mathcal{M}_{i}$. At stages $s$ when $\mathcal{N}_{k}$ is following $\mathcal{M}_{i}$ we construct $\mathcal{N}_{k, s}$ to be isomorphic to $\mathcal{M}_{i}\left\lceil s\right.$. If at any point we release $\mathcal{N}_{k}$ as a follower of $\mathcal{M}_{i}$, we call $\mathcal{N}_{k}$ free and $\mathcal{N}_{k}$ will never again be assigned to follow any other $\mathcal{M}_{j}$. However, $\mathcal{M}_{i}$ can be assigned a new follower at a later stage. By Assumption (1a), for all $j \in \omega$, the structure $\mathcal{M}_{j}\lceil 0$ generated by the empty set lies in $\mathcal{K}$; we consider in Corollary 2.7 below how to amend this assumption. At each stage $s$, we take action for some $\mathcal{M}_{i}$. We let $e_{s}$ denote the index $i$ of the $\mathcal{M}_{i}$ for which we take action at stage $s$. Specifically, we set $e_{s}$ equal to the number of prime factors of $s$. This definition ensures that we take action for each $\mathcal{M}_{e}$ at infinitely many stages during the construction.

Assumptions (2a) and (2b) imply that $\mathcal{M}_{i}$ and $\mathcal{M}_{j}$ being isomorphic is a $\Pi_{2}^{d}$-property. In particular, we may define a $\boldsymbol{d}$-computable chip function $c(i, j, s)$ as follows:

$$
c(i, j, s)= \begin{cases}0 & \text { if } s=0 . \\ c(i, j, s-1)+1 & \text { if } \mathcal{M}_{i} \uparrow t \text { embeds into } \mathcal{M}_{j} \uparrow s \text { and } \\ & \mathcal{M}_{j}\left\lceil t \text { embeds into } \mathcal{M}_{i} \uparrow s,\right. \\ & \text { where } t=c(i, j, s-1), s>0 . \\ c(i, j, s-1) & \text { otherwise. }\end{cases}
$$

In other words, $c(i, j, s)$ "gives a chip" to the pair $(i, j)$ at stage $s$ (i.e. outputs $c(i, j, s)=c(i, j, s-1)+1)$ if and only if the stage $t$ approximations of $\mathcal{M}_{i}$ and $\mathcal{M}_{j}$ embed into each other's stage $s$ approximations, where $t$ is the total number of chips received by the pair $(i, j)$ at all stages less than $s$. This definition is symmetric in $i$ and $j$, and the pair $(i, j)$ receives infinitely many chips (over all stages $s$ ) if and only if $\mathcal{M}_{i} \cong \mathcal{M}_{j}$.

We will see by induction that the construction satisfies the following assumption: that for each stage $t<s$ and each $\mathcal{N}_{i}$ (which may be a follower or free at stage $t$ ), we $\boldsymbol{d}$-computably know a $\boldsymbol{d}$-computable index $e^{\prime}$ and stage $t^{\prime}$ such that $\mathcal{N}_{i, t} \cong \mathcal{M}_{e^{\prime}} \mid t^{\prime}$. Hence, by Assumption (2b), it is $\boldsymbol{d}$-computable to determine whether a given $\mathcal{N}_{i, t}$ is isomorphic to a given $\left.\mathcal{M}_{\hat{e}}\right\rceil \hat{t}$. We may also inductively assume that the current follower $\mathcal{N}_{k}$ for $\mathcal{M}_{e_{s}}$ at the beginning of stage $s$, if any, satisfies $\mathcal{N}_{k, t}=\mathcal{N}_{k, s-1} \cong \mathcal{M}_{e_{s}}\lceil t$ for some $t<s$. (In other words, $\mathcal{N}_{k}$ has not changed since stage $t$.)

At stage $s$, having fixed $e_{s}$, we have three cases.

Case $1\left(\mathcal{M}_{e_{s}}\right.$ with follower appears isomorphic to earlier $\left.\mathcal{M}_{e}.\right)$ Suppose that $\mathcal{M}_{e_{s}}$ has a follower $\mathcal{N}_{k}$ and that there exists an $e<e_{s}$ with $c\left(e, e_{s}, s\right) \geq k$. Then we release $\mathcal{N}_{k}$ as a follower of $\mathcal{M}_{e_{s}}$.

Case 2 (For some $k$ with additional properties, $\left.\mathcal{N}_{k, s-1} \cong \mathcal{M}_{e_{s}}\right\rceil s$.) 
If Case 1 does not hold, and there exists a $k$ such that $\mathcal{N}_{k, s-1} \cong \mathcal{M}_{e_{s}}\lceil s$ with one of the following properties:

- $\mathcal{N}_{k}$ is the follower of $\mathcal{M}_{e}$ for some $e \leq e_{s}$; or

- $\mathcal{N}_{k}$ is not currently a follower of any $\mathcal{M}_{e}$, and either $k \leq e_{s}$ or $\mathcal{N}_{k}$ was previously displaced by $\mathcal{M}_{e_{s}}$ via Case 3,

then we do nothing.

Case 3 (Case 1 and Case 2 do not hold.). If Case 1 and Case 2 do not hold, we execute the following three steps.

1. (Ensure $\mathcal{M}_{e_{s}}$ has a follower.)

If $\mathcal{M}_{e_{s}}$ has no follower, assign $\mathcal{N}_{k}$ to follow $\mathcal{M}_{e_{s}}$ where $k$ is the least index for which $\mathcal{N}_{k}$ has never yet been a follower, and build $\mathcal{N}_{k, s}$ isomorphic to $\mathcal{M}_{e_{s}}\lceil s$.

2. (Update any existing follower for $\mathcal{M}_{e_{s}}$.)

If $\mathcal{M}_{e_{s}}$ already had a follower $\mathcal{N}_{k}$ at stage $(s-1)$, then add elements to $\mathcal{N}_{k, s-1}$ as needed so that $\mathcal{N}_{k, s} \cong \mathcal{M}_{e_{s}}\lceil s$. (This is possible by our second inductive hypothesis. Specifically, $\mathcal{N}_{k}$ satisfied $\left.\mathcal{N}_{k, t} \cong \mathcal{M}_{e_{s}}\right\rceil t$ at the most recent stage $t<s$ with $e_{t}=e_{s}$ and has not changed since then.)

Steps 1 and 2 together ensure that $\mathcal{N}_{k, s} \cong \mathcal{M}_{e_{s}}\lceil s$, no matter whether $\mathcal{N}_{k}$ was previously a follower of $\mathcal{M}_{e}$ or not.

3. (Some $\mathcal{N}_{k^{\prime}, s-1}$ besides $\mathcal{M}_{e_{s}}$ 's follower is isomorphic to $\left.\mathcal{M}_{e_{s}}\right\rceil s$. )

Suppose there is some $k^{\prime} \neq k$ such that $\mathcal{N}_{k^{\prime}, s-1} \cong \mathcal{M}_{e_{s}}\lceil s$. In this case, we release this $k^{\prime}$ from being the follower of any $\mathcal{M}_{e^{\prime}}$ for which it was a follower at stage $(s-1)$, and (whether it was released here or previously) we say that $k^{\prime}$ has been displaced by $\mathcal{M}_{e_{s}}$ at this stage. Since Case 2 did not apply, if $\mathcal{N}_{k^{\prime}}$ is was a follower of some $\mathcal{M}_{e^{\prime}}$ at this stage, then $e^{\prime}>e_{s}$; while, if not, then $k^{\prime}>e_{s}$ and this is the first time $k^{\prime}$ has been displaced by $\mathcal{M}_{e_{s}}$. By Assumption (1b), there is a stage $t^{\prime}>s$ and some $\mathcal{M}_{j}$, which we can find with our $\boldsymbol{d}$-oracle, such that:

- $\left.\mathcal{N}_{k^{\prime}, s-1} \cong \mathcal{M}_{e_{s}}\right\rceil s$ is a proper substructure of $\mathcal{M}_{j} \backslash t^{\prime}$ and

- for all $i<s, \mathcal{M}_{j}\left\lceil t^{\prime}\right.$ is not isomorphic to $\mathcal{M}_{i}\lceil s$.

We add elements to $\mathcal{N}_{k^{\prime}}$ to make $\mathcal{N}_{k^{\prime}, s} \cong \mathcal{M}_{j} \mid t^{\prime}$.

(If there were more than one such $k^{\prime} \neq k$, these instructions for Step 3 would have us repeat the process again for each such $k^{\prime}$. In fact, though, this step ensures that all $\mathcal{N}_{k, s}$ are pairwise nonisomorphic, for all $k$ which have been chosen as followers up to this stage. So, by induction on the preceding stages, there will be at most one such $k^{\prime}$. The induction continues since Case 3 is the only case that changes followers and Step 3 ensures $\mathcal{N}_{k, s} \neq \mathcal{N}_{k^{\prime}, s}$.)

This ends stage $s$, and the construction is now complete. Also, the inductive hypotheses stated earlier are now clear.

We follow Friedberg's argument to show that the $\boldsymbol{d}$-computable enumeration $\left\langle\mathcal{N}_{i}\right\rangle_{i \in \omega}$ thus produced is in fact a classification of the entire family $\mathcal{K}$ of structures. Clearly it is a $\boldsymbol{d}$-computable enumeration of structures.

Lemma 2.4 If $\mathcal{M}_{e} \neq \mathcal{M}_{i}$ for all $i<e$, then there exists some $k$ with $\mathcal{N}_{k} \cong \mathcal{M}_{e}$. 
Proof. We follow Friedberg [8, Lemma 3, p. 313]. Fix $c=\max _{i<e} \lim _{s} c(e, i, s)$, which must be finite. Then no follower $\mathcal{N}_{k}$ of $\mathcal{M}_{e}$ with $k>c$ will ever be released by the action of Step 1, and so there are only finitely many stages at which $\mathcal{M}_{e}$ loses a follower in this way. A follower of $\mathcal{M}_{e}$ may also be released by the action of Case 3 for some $e_{s}<e$. We claim that there are only finitely many stages $s$ at which a follower of $\mathcal{M}_{e}$ is released by the action of Case 3 for any $e_{s}<e$. If not, there are infinitely many such stages $t_{0}<t_{1}<t_{2}<\ldots$ for a single $e^{\prime}=e_{s}<e$. For all $i \in \omega$, let $\mathcal{N}_{k_{i}}$ be the follower of $\mathcal{M}_{e}$ at stage $t_{i}-1$ such that $\mathcal{N}_{k_{i}, t_{i}-1} \cong \mathcal{M}_{e^{\prime}}\left\lceil t_{i}\right.$. Since $\mathcal{N}_{k_{i}, t_{i}-1}$ is a follower of $\mathcal{M}_{e}$, we have that $\left.\mathcal{N}_{k_{i}, t_{i}-1} \cong \mathcal{M}_{e}\right\rceil \tilde{t}_{i}$ for some $\tilde{t}_{i}$ satisfying $t_{i-1}<\tilde{t}_{i}<t_{i}$ for all $i>0$. Thus, $\mathcal{M}_{e^{\prime}}\left\lceil t_{i} \cong \mathcal{M}_{e}\left\lceil\tilde{t}_{i}\right.\right.$ for all $i>0$, and $\mathcal{M}_{e} \cong \mathcal{M}_{e^{\prime}}$ by Assumption (2a). This contradicts the lemma's hypothesis, so there is some stage $s_{0}$ after which $\mathcal{M}_{e}$ never loses a follower. If at any stage $s>s_{0}$ with $e_{s}=e$ we reach Case 3 , then $\mathcal{M}_{e}$ will thereafter have a follower $k$ which it never loses. From then on, whenever $\mathcal{M}_{e} \uparrow(t+1) \neq \mathcal{M}_{e}\lceil t$, if Case 3 applies at the next stage $s>t$ with $e_{s}=e$, Step 2 of Case 3 will add elements to $\mathcal{N}_{k}$ to make $\mathcal{N}_{k, s} \cong M_{e}\lceil s$ again, whereas no other elements will ever be added to $\mathcal{N}_{k}$ at any other stage. Thus, if Case 3 occurs infinitely often with $e_{s}=e$, then $\mathcal{N}_{k} \cong \mathcal{M}_{e}$.

If Case 3 occurs only finitely often with $e_{s}=e$, then Case 2 occurs at infinitely many stages instead. (Case 1 would cause $\mathcal{N}_{k}$ to be released, which will never happen.) At each such stage, there is some $k^{\prime}$ with $\mathcal{N}_{k^{\prime}, s-1} \cong \mathcal{M}_{e}\lceil s$, satisfying one of the disjuncts of Case 2 . In particular, either $\mathcal{N}_{k^{\prime}}$ is a follower of some $\mathcal{M}_{i}$ with $i<e, \mathcal{N}_{k^{\prime}}$ is not a follower and $k^{\prime} \leq e$, or $\mathcal{M}_{e}$ previously displaced $\mathcal{N}_{k^{\prime}}$ in Case 3 . We first argue that there are only finitely many $k^{\prime}$ such that $\mathcal{N}_{k^{\prime}, s-1} \cong \mathcal{M}_{e}\left\lceil s\right.$ is a follower of some $\mathcal{M}_{i}$ with $i<e$ at the stages when Case 2 occurs with $e_{s}=e$. Suppose otherwise. Let $e^{\prime}<e$ be the least index for which there are infinitely many such $k^{\prime}$. Consider $k_{0}<k_{1}<k_{2}<\ldots$ and stages $t_{0}<t_{1}<t_{2}<\ldots$ after $s_{0}$ where $\mathcal{N}_{k_{i}}$ is following $\mathcal{M}_{e^{\prime}}$ at stage $t_{i}$, $e_{t_{i}}=e$, and $\mathcal{N}_{k_{i}, t_{i}-1} \cong \mathcal{M}_{e}\left\lceil t_{i}\right.$. Now, $\mathcal{N}_{k_{i}, t_{i}-1} \cong \mathcal{M}_{e^{\prime}} \mid \tilde{t}_{i}$ for some $\tilde{t}_{i}$ such that $t_{i-1}<\tilde{t}_{i}<t_{i}$ for $i>0$. Thus, $\mathcal{M}_{e^{\prime}} \mid \tilde{t}_{i} \cong \mathcal{N}_{k_{i}, t_{i}-1} \cong \mathcal{M}_{e}\left\lceil t_{i}\right.$ for all $i>0$. By Assumption (2a), we have $\mathcal{M}_{e^{\prime}} \cong M_{e}$, contradicting the lemma's hypothesis. By the above claim and the fact that $\mathcal{M}_{e}$ only executed Case 3 at finitely many stages, there are only finitely many $k^{\prime}$ for which any of these conditions could hold. Therefore, one of those $k^{\prime}$ satisfies $\mathcal{N}_{k^{\prime}, s-1} \cong \mathcal{M}_{e}\lceil s$ at infinitely many stages $s$, and therefore $\mathcal{N}_{k^{\prime}} \cong \mathcal{M}_{e}$.

Lemma 2.4 and the construction now imply that every $\mathcal{N}_{k}$ eventually becomes a follower of an $\mathcal{M}_{e}$, at least temporarily, just as shown in the proof in [8]. We also now imitate Lemmas 4 and 5 from that proof ([8, p. 315]). We say that $\mathcal{N}_{k}$ is finitely generated if there exists some $t$ such that $\mathcal{N}_{k, t}=\mathcal{N}_{k}$, i.e. $\mathcal{N}_{k}=\mathcal{M}_{j}\lceil s$ for some $j, s \in \omega$.

Lemma 2.5 If $k \neq k^{\prime}$ and $\mathcal{N}_{k}$ and $\mathcal{N}_{k^{\prime}}$ are both finitely generated, then $\mathcal{N}_{k} \neq \mathcal{N}_{k^{\prime}}$.

Proof. By assumption, there exist $t$ and $t^{\prime}$ such that $\mathcal{N}_{k, t}=\mathcal{N}_{k}$ and $\mathcal{N}_{k^{\prime}, t^{\prime}}=\mathcal{N}_{k^{\prime}}$. Moreover, we saw above that each must eventually become a follower, say of $\mathcal{M}_{e}$ and $\mathcal{M}_{e^{\prime}}$, respectively. Now consider the first stage $s$ such that $\mathcal{N}_{k}=\mathcal{N}_{k, s} \cong \mathcal{N}_{k^{\prime}, s}=\mathcal{N}_{k^{\prime}}$ (and such that $k$ and $k^{\prime}$ have both become followers by stage $s$ ). Either one of $k$ and $k^{\prime}$ became a follower at this stage, 
or else the congruence arose because elements were added to one of $\mathcal{N}_{k}$ or $\mathcal{N}_{k^{\prime}}$ at this stage. Therefore, we must been in Case 3 at stage $s$, and will have executed Step 3 at this stage. Without loss of generality assume that $e_{s}=e$. Then steps 1 and 2 ensured that $\mathcal{N}_{k, s} \cong \mathcal{M}_{e}\left\lceil s\right.$. If $\mathcal{N}_{k^{\prime}, s-1} \not \mathcal{M}_{e}\lceil s$, then no elements would have been added to $\mathcal{N}_{k^{\prime}}$ at stage $s$, contradicting $\mathcal{N}_{k^{\prime}, s} \cong \mathcal{N}_{k, s}$. Therefore, $\mathcal{N}_{k^{\prime}, s-1} \cong \mathcal{M}_{e}\left\lceil s\right.$, so we executed Step 3 for this $k^{\prime}$, placing new elements in $\mathcal{N}_{k, s}$ so that $\mathcal{N}_{k^{\prime}, s} \cong \mathcal{M}_{j}\left\lceil t^{\prime} \neq M_{e}\lceil s\right.$, using the $j$ and $t^{\prime}$ found at that step. Thus $\mathcal{N}_{k^{\prime}, s} \neq \mathcal{N}_{k, s}$, contradicting our choice above of the stage $s$. So in fact $\mathcal{N}_{k} \neq \mathcal{N}_{k^{\prime}}$.

Lemma 2.6 If $k \neq k^{\prime}$ and neither $\mathcal{N}_{k}$ nor $\mathcal{N}_{k^{\prime}}$ is finitely generated, then $\mathcal{N}_{k} \neq \mathcal{N}_{k^{\prime}}$.

Proof. Every $\mathcal{N}_{k}$ eventually becomes a follower of some $\mathcal{M}_{e}$. If it is later released by $\mathcal{M}_{e}$, then thereafter it is never again a follower, and may be displaced at most once by each $\mathcal{M}_{e^{\prime}}$ with $e^{\prime}<x$ and never by any other $\mathcal{M}_{e^{\prime}}$. Hence it is modified only finitely often in all, leaving it finitely generated. Thus we may assume that neither of $\mathcal{N}_{k}$ and $\mathcal{N}_{k^{\prime}}$ is ever released.

Suppose $\mathcal{N}_{k} \cong \mathcal{N}_{k^{\prime}}$, and say that they are followers of $\mathcal{M}_{e}$ and $\mathcal{M}_{e^{\prime}}$, respectively. Without loss of generality, take $e<e^{\prime}$. ( $\mathcal{M}_{e}$ can have at most one follower which it never releases, so with $k \neq k^{\prime}$, we have $e \neq e^{\prime}$.) Moreover, in order not to be finitely generated, $\mathcal{N}_{k}$ must undergo Step 2 in Case 3 infinitely often, as must $\mathcal{N}_{k^{\prime}}$, and therefore $\mathcal{M}_{e} \cong \mathcal{N}_{k} \cong \mathcal{N}_{k^{\prime}} \cong \mathcal{M}_{e^{\prime}}$. But then $c\left(e, e^{\prime}, s\right) \rightarrow \infty$ as $s \rightarrow \infty$, so there must exist a stage $s$ with $e_{s}=e^{\prime}$ at which $c\left(e, e^{\prime}, s\right) \geq k^{\prime}$, and at this stage Case 1 will cause $\mathcal{N}_{k^{\prime}}$ to be released as a follower of $\mathcal{M}_{e^{\prime}}$, yielding a contradiction.

Of course, $\mathcal{N}_{k} \neq \mathcal{N}_{k^{\prime}}$ whenever just one of $\mathcal{N}_{k}$ and $\mathcal{N}_{k^{\prime}}$ is finitely generated, and so the two preceding lemmas show $\left\langle\mathcal{N}_{k}\right\rangle_{k \in \omega}$ to be one-to-one up to isomorphism. Lemma 2.4 then shows it to be a $\boldsymbol{d}$-computable classification of $\mathcal{K}$ by $\boldsymbol{d}$-computable indices.

To see that Theorem 2.3 applies to the family $\mathcal{K}$ of all computable algebraic field extensions of the prime field $Q$ of characteristic $p$, we simply use the facts already stated about such fields. Lemma 2.1 gives a computable enumeration of $\mathcal{K}$. Every subfield of a field in $\mathcal{K}$ is also in $\mathcal{K}$, so Assumption (1a) holds. For Assumption (1b), given the finitely generated fields $\mathcal{M}_{k}\lceil s$ for all $k<s$, fix some prime number $d \neq p$ which is greater than the dimension of each of these fields over $Q$, and adjoin a $d$-th root of unity to $\mathcal{M}_{i}\lceil s$ to get a computably presentable, finitely generated subfield of $\bar{Q}$. Some $\mathcal{M}_{j}$ in our enumeration of fields must be isomorphic to this subfield, and some $t$ satisfies $\mathcal{M}_{j} \uparrow t=\mathcal{M}_{j}$ (in fact, $j$ and $t$ can be found effectively), but by the choice of $d$, we know that $\mathcal{M}_{k}\left\lceil s \neq \mathcal{M}_{j} \uparrow t\right.$. Finally, the assumptions (2a) and (2b) are both standard for algebraic fields. Lemma 2.2 establishes (2a). For (2b), we appeal to Kronecker's Theorem, from [14], as given in [15, Theorem $2.2]$, for example: it states that we have splitting algorithms for every finitely generated subfield $F$ of $\bar{Q}$, uniformly in the generators of the subfield. This means that, given any $x \in \bar{Q}$, one can find the minimal polynomial $f(X)$ of $x$ over $Q$ and factor $f(X)$ in $F[X]$ effectively; then $f$ has a root in $F$ if and only if at least one of its factors in $F[X]$ is linear. Given $F_{i}$ and $F_{j}$, we can find a primitive generator $x$ for $F_{i}$ and execute this process. Now $F_{i}$ embeds 
into $F_{j}$ if and only if the minimal polynomial of $x$ over $Q$ has a root in $F_{j}$, so the splitting algorithm for $F_{j}$ tells us whether $F_{i}$ embeds into $F_{j}$. Moreover, $F_{i} \cong F_{j}$ if and only if each embeds into the other, so we have a decision procedure for deciding isomorphism as well. This is all that is required by Theorem 2.3, so we have proven the existence of a computable classification of all computable algebraic fields of any fixed charateristic. Finally, our proof is uniform in the characteristic, and hence also yields a computable classification of all computable algebraic fields, as claimed in Theorem 1.9.

Theorem 2.3 works well for families of structures, such as fields of a given characteristic, which have a prime model. The prime model is analogous to the empty set in the original Friedberg construction. Since our theorem requires that every $\mathcal{M}_{i}\lceil 0$ lie in the family $\mathcal{K}$, however, it is awkward to apply it to families with no prime model. One solution is to consider the empty structure as an element of such a family. In general, though, this difficulty can be avoided by a slight modification to the proof of the theorem.

Corollary 2.7 Let $\boldsymbol{d}, \mathcal{K}$, and $\left\langle\mathcal{M}_{i}\right\rangle_{i \in \omega}$ satisfy all the hypotheses of Theorem 2.3, except that in Assumption (1a), we only require that each $\mathcal{M}_{i} \uparrow(s+1)$ lie in $\mathcal{K}$. Then the conclusion still holds: there exists a $\boldsymbol{d}$-computable classification of $\mathcal{K}$ by $\boldsymbol{d}$-computable indices.

Proof. We simply regard every $\mathcal{M}_{i} \uparrow 0$ as empty, and likewise regard $\mathcal{N}_{k, s}$ as empty for every stage $s$ at which $\mathcal{N}_{k}$ has not yet been chosen as a follower of any $\mathcal{M}_{e}$. Every $\mathcal{N}_{k}$ is eventually chosen as a follower, and when it is (in Case 3 , at some stage $s>0$ ), the construction sets $\mathcal{N}_{k, s} \cong \mathcal{M}_{e_{s}}\lceil s$, which lies in $\mathcal{K}$. Therefore, no $\mathcal{N}_{k}$ winds up empty, and the rest of the proof proceeds exactly as for Theorem 2.3.

\section{Torsion-Free Abelian Groups of Rank 1}

The construction of Theorem 2.3 does not apply to the family $\mathcal{T}$ of computable finite-branching trees, nor to the family $\mathbf{T F A} \mathbf{b}_{1}$ of torsion-free abelian groups of rank 1 , and its failure to do so demonstrates the sharpness of the conditions given in the theorem. For the trees, we will see in Proposition 4.2 that there is no computable enumeration of the computable finite-branching trees (analogous to $\left\{F_{n}: n \in \omega\right\}$ above for fields), so there is certainly no computable classification, even though the other hypotheses of Theorem 2.3 hold. (In particular, the isomorphism problem is exactly the same as for algebraic fields.) For $\mathbf{T F A b} \mathbf{b}_{1}$, Proposition 3.1 gives a computable enumeration, yet Corollary 3.3 below implies that there is no computable classification of $\mathbf{T F A} \mathbf{b}_{1}$. Here Theorem 2.3 does not apply since the isomorphism problem is no longer $\Pi_{2}^{0}$ (see Lemma 3.2): we do not have any nice way of comparing two such groups and guessing whether they are isomorphic. In this section we prove these results for $\mathbf{T F A b}_{1}$.

Proposition 3.1 There is a computable enumeration of the family $\boldsymbol{T F A \boldsymbol { b } _ { 1 }}$ of all computable torsion-free abelian groups of rank 1 .

Proof. The proof is similar to the argument in Lemma 2.1 for algebraic fields. We fix a computable presentation of the additive group $(\mathbb{Q}, 0,+)$ and list out the subgroups generated by each c.e. subset $W_{e}$ of its domain. 
The following lemma of Calvert [2, Theorem 2.4.3] established that the isomorphism problem for torsion-free abelian groups of any fixed finite rank is $\Sigma_{3}^{0}$-complete.

Lemma 3.2 (Calvert) The isomorphism problem $E$ for $\mathbf{T F A b _ { 1 }}$ is $\Sigma_{3}^{0}$ complete under m-reducibility.

For the next Corollary, it may be useful to review Definition 1.7.

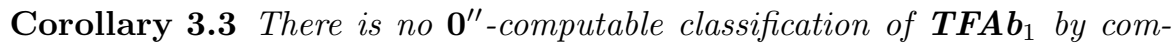
putable indices.

Proof. To prove this, we establish a strong version of the second part of Proposition 1.5, stating that, for each $n \in \omega$, no family $\mathcal{K}$ of computable structures, closed under isomorphism, whose isomorphism problem is $\Sigma_{n}^{0}$-complete and whose index set is $\Delta_{n}^{0}$ has a $\mathbf{0}^{(n-1)}$-computable classification by computable indices.

Suppose that $f$ were a $\mathbf{0}^{(n-1)}$-computable total function classifying $\mathcal{K}$ by computable indices. Then, with a $\mathbf{0}^{(n-1)}$-oracle, we could decide the isomorphism problem $E$ for $\mathcal{K}$ as follows. Given indices $i$ and $j$, first use the oracle to check whether they are both indices of elements of $\mathcal{K}$. If so, then use the oracle to enumerate the $\Sigma_{n}^{0}$ set $E$ until we find numbers $a$ and $b$ such that $(i, f(a)) \in E$ and $(j, f(b)) \in E$. This must happen, because the image of the classification $f$ contains an index for a computable copy of each computable torsion-free abelian group. However, because the image contains only one such index for each such group, we know that $(f(a), f(b)) \in E$ iff $a=b$. Therefore, $(i, j) \in E$ iff $a=b$, Hence, $E$ would be a $\Delta_{n}^{0}$ set, a contradiction.

Since the index set of $\mathbf{T F A} \mathbf{b}_{1}$ is $\Pi_{2}^{0}$ and hence $\Delta_{3}^{0}$, Lemma 3.2 then establishes Corollary 3.3.

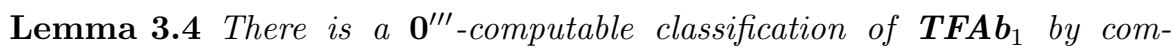
putable indices.

Proof. It is simple to construct such a $\mathbf{0}^{\prime \prime \prime}$-computable classification $g$. A $\mathbf{0}^{\prime \prime \prime}$ oracle can decide both the index set $I$ for $\mathbf{T F A} \mathbf{b}_{1}$ and its isomorphism problem $E$, since these are $\Pi_{2}^{0}$ and $\Sigma_{3}^{0}$, respectively. So let $g(0)$ be the least element of $I$, and for each $n$, let $g(n+1)$ be the least element $j \in I$ with $j>g(n)$ and $(\forall i<j)(i, j) \notin E$. This suffices. (Indeed, since $g$ is an increasing function, its image is also $\mathbf{0}^{\prime \prime \prime}$-decidable.)

Lemma 3.4 and Corollary 3.3 together prove Theorem 1.10. We are left with the following natural question, which is answered elsewhere in this article for all other structures we consider, but remains open for $\mathbf{T F A \mathbf { b } _ { 1 }}$.

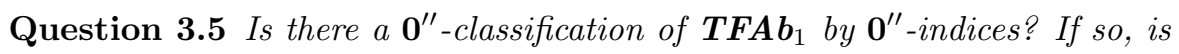
there a $\mathbf{0}^{\prime}$-classification by $\mathbf{0}^{\prime}$-indices?

We draw attention to the contrast between Theorem 1.9 and Theorem 1.10. Algebraic fields and rank-1 torsion-free abelian groups are usually regarded as highly similar families of structures. In each case, every element $x$ of a computable model of the structure can be identified effectively up to finitely many possibilities: in fields, finding the minimal polynomial of $x$ over the prime subfield accomplishes this, while in groups, having fixed a single nonidentity element $z$, one finds a nontrivial relation on $z$ and $x$, expressed as $x=q z$ for some $q \in \mathbb{Q}$. Such a relation must exist, since the group has rank 1 , 
and once it is found, $x$ is known to be the unique element satisfying it, since the group is isomorphic to an additive subgroup of $\mathbb{Q}$. One might suspect that therefore the groups would be more amenable to classification than the fields, at least given finitely much information (namely the parameter $z$ ). Theorems 1.9 and 1.10 reverse this intuition.

Moreover, in computable structure theory, it is known that these two families have exactly the same possible spectra. Recall the relevant definition.

Definition 3.6 For a countable structure $\mathcal{A}$, the spectrum of $\mathcal{A}$ is the set of all Turing degrees of structures isomorphic to $\mathcal{A}$ :

$$
\operatorname{Spec}(\mathcal{A})=\{\operatorname{deg}(\mathcal{B}): \mathcal{B} \cong \mathcal{A} \& \operatorname{dom}(\mathcal{B})=\omega\} .
$$

(We consider only structures $\mathcal{B}$ with domain $\omega$, so the degree of $\mathcal{B}$ is always a well-defined concept.)

The following result was proven for $\mathbf{T F A} \mathbf{b}_{1}$ by Coles, Downey, and Slaman in [6], and for algebraic fields by Frolov, Kalimullin, and Miller in [9].

Theorem 3.7 For every set $\mathcal{U}$ of Turing degrees, the following are equivalent.

- $\mathcal{U}$ is the spectrum of some infinite algebraic field.

- $\mathcal{U}$ is the spectrum of some torsion-free abelian group of rank 1.

- There exists a set $U \subseteq \omega$ for which

$$
\mathcal{U}=\{\boldsymbol{d}: U \text { is } \boldsymbol{d} \text {-computably enumerable }\} .
$$

However, despite the similarities between the families of algebraic fields and torsion-free abelian groups, their classification problems (for computable structures) turned out to be of significantly different complexity: we found a computable classification of all computable algebraic fields, whereas, using computable indices, $\mathbf{T F A} \mathbf{b}_{1}$ has only a $\mathbf{0}^{\prime \prime \prime}$-computable classification.

\section{Finite-Branching Trees}

Recall that $\mathcal{T}$ is the class of all computable finite-branching trees, under the function $P$ which maps each node in a tree to its immediate predecessor. (By convention, $P$ maps the root of a tree to itself.) It is often simplest to view a finite-branching tree just as a tree in which each level has only finitely many nodes. Nevertheless, the usual definition of finite-branching (that each node has only finitely many immediate successors) has the least possible complexity, as we now show.

Lemma 4.1 The index set I for the family $\mathcal{T}$ is $\Pi_{3}^{0}$-complete.

Proof. To see that $I$ is $\Pi_{3}^{0}$, notice that the partial computable function $\varphi_{e}$ is the predecessor function for a finite-branching tree with domain $\omega$ if and only if the following all hold.

- $\varphi_{e}$ is total.

- There is a unique $r$ for which $\varphi_{e}(r)=r$.

- For every $x \in \omega$, there exists an $l$ such that $\varphi_{e}^{l}(x)=\varphi_{e}^{l+1}(x)$. (This means that, for the least such $l$, the $l$-th predecessor $\varphi_{e}^{l}(x)$ of $x$ is the root, so that $x$ lies at the level $l$ of the tree.)

- For every $l \in \omega$, there are only finitely many $x \in \omega$ with $\varphi_{e}^{l}(x)=\varphi_{e}^{l+1}(x)$. (This says that the tree has only finitely many nodes at each level $l$, which is equivalent to being finite-branching.) 
One can also check, using only a $\mathbf{0}^{\prime \prime}$-oracle, whether $\varphi_{e}$ has finite domain and computes a tree on that domain. Therefore the set $I$ (even including indices of finite trees) is $\Pi_{3}^{0}$.

To show that $I$ is $\Pi_{3}^{0}$-complete under $m$-reducibility, we give an $m$-reduction from the complement of Cof to $I$. Given any index $e \in \omega$, build the computable tree $T_{e}$ with root 0 as follows. At stage $s$, let the least fresh element of $\omega$ lie at level $s+1$ in $T_{e}$, with the least node at level $s$ as its immediate predecessor. Then, if the $n$-th smallest element of the complement of $W_{e, s}$ lies in the set $W_{e, s+1}$, add a new node to level $n+1$ of $T_{e}$, with the least node at level $n$ as its immediate predecessor. This is the entire construction. If $e \in \mathbf{C o f}$, then for $n=\left|\overline{W_{e}}\right|$, the $(n+1)$-st smallest element of $W_{e, s}$ entered $W_{e, s+1}$ at infinitely many stages $s$, and therefore $T_{e}$ is infinite-branching, with infinitely many nodes at level $(n+2)$. On the other hand, if $e \notin \mathbf{C o f}$, then for every $n$, the $(n+1)$-st level of $T_{e}$ only received a new node at finitely many stages, and so $T_{e}$ is finite-branching. Thus we have the necessary $m$-reduction.

Proposition 4.2 There is no $\mathbf{0}^{\prime \prime}$-computable enumeration of all computable finite-branching trees by computable indices.

Proof. First note that the isomorphism problem $E$ for computable finitebranching trees is $\Pi_{2}^{0}$, since two finite-branching trees are isomorphic if and only if every finite subtree of each one embeds into the other. (For details, see [19].) Moreover, the same statement holds of any two computable trees under predecessor, provided only that at least one of them is finite-branching. Suppose $S$ is a finite-branching tree and $T$ is an infinite-branching tree. Then we can choose the least infinite-branching node $x \in T$, say at level $l$, and consider the finite subtree consisting of $x$, its predecessors, and $(a+1)$ of its immediate successors, where $a$ is the number of nodes at level $(l+1)$ in the finite-branching tree $S$. Clearly this finite subtree of $T$ cannot embed into $S$ (recalling that an embedding must map the root to the root), and so the $\Pi_{2}^{0}$ condition fails for this pair $(S, T)$. (The $\Pi_{2}^{0}$ condition can hold for non-isomorphic $S$ and $T$ when both are infinite-branching.)

With this information we can show that the existence of such an enumeration $f$ would force the index set $I$ of the family of computable finite-branching trees to be $\Sigma_{3}^{0}$. Indeed, an index $e$ would lie in $I$ if and only if $\varphi_{e}$ computes a tree $T_{e}$ under predecessor, either with domain $\omega$ or with finite domain (all of which is $\mathbf{0}^{\prime \prime}$-decidable), and there exists some $n \in \omega$ such that every finite subtree of each of $T_{e}$ and $T_{f(n)}$ embeds into the other (which is $\Sigma_{3}^{0}$, including the quantifier $(\exists n)$ ). Indeed, since $T_{f(n)}$ is known to be finite-branching, the preceding paragraph shows that $T_{e} \cong T_{f(n)}$ if and only if the $\Pi_{2}^{0}$ condition on embedding of finite subtrees holds; conversely, if $T_{e}$ really is finite-branching, then it must be isomorphic to some $T_{f(n)}$. Thus $I$ would be $\Sigma_{3}^{0}$, contrary to Lemma 4.1.

Theorem 1.11 follows from Proposition 4.2 along with the next results.

Proposition 4.3 There exists a $\mathbf{0}^{\prime \prime \prime}$-computable classification of all computable finite-branching trees by computable indices.

Proof. The $\mathbf{0}^{\prime \prime \prime}$-classification $f$ is readily given: $f(n)$ is the least $m>f(n-1)$ (or the least $m \geq 0$, if $n=0$ ) such that $m$ lies in the index set for computable finite-branching trees and, for all $k<n$, the tree $T$ computed by $\varphi_{m}$ is 
not isomorphic to that computed by $\varphi_{f(k)}$. Lemma 4.1 shows that the first part of this is a $\Pi_{3}^{0}$ condition, hence decidable by our $\mathbf{0}^{\prime \prime \prime}$-oracle, and the isomorphism problem for these trees is $\Pi_{2}^{0}$, as discussed in Proposition 4.2. In fact, the image of this classification function $f$ is $\Delta_{4}^{0}$, since $f$ itself is strictly increasing.

Finally, we apply Friedberg's method (as adapted in Theorem 2.3) to give a simpler classification of the finite-branching computable trees: this classification requires only a $\mathbf{0}^{\prime}$-oracle, but uses $\mathbf{0}^{\prime}$-computable indices.

Proposition 4.4 There exists a $\mathbf{0}^{\prime}$-computable classification of the family $\mathcal{T}$ of all computably presentable finite-branching trees by $\mathbf{0}^{\prime}$-computable indices.

Proof. In order to fulfill the hypotheses of Theorem 2.3, we first give a $\mathbf{0}^{\prime}$ computable enumeration $g$ of $\mathcal{T}$ by $\mathbf{0}^{\prime}$-computable indices. For each $e$, let $g(e)$ be an index for the $\mathbf{0}^{\prime}$-computable tree $T_{e}$ defined as follows, with root 0 . Use the oracle to ask whether $\varphi_{e}$ has a fixed point $r$, and, if so, whether it is unique (i.e. ask whether $\forall x \forall s\left(\varphi_{e, s}(x) \downarrow=x \Longrightarrow x=r\right)$ ). If not, then $T_{e}$ consists just of its root 0 . If so, then, using the oracle, check that $\varphi_{e}(0) \downarrow$, and search for an $m$ such that no element greater than $m$ has $r$ as a predecessor (i.e. ask whether $\left.(\forall s \forall x>m)\left(\neg \varphi_{e, s}(x) \downarrow=r\right)\right)$. If $\varphi_{e}(0) \uparrow$, or if we never find such an $m$, then again $T_{e}$ will consist only of the root. Otherwise, having found $m$, we add one node at level one to $T_{e}$ for each $x \leq m$ with $x \neq r$ and $\varphi_{e}(x) \downarrow=r$ (which our oracle can check). Thus we have a one-to-one correspondence between the nodes at level 1 in $T_{e}$ and those at level 1 in the tree $S_{e}$ (if any) computed by $\varphi_{e}$, provided that $S_{e}$ is finite-branching at its root. This completes level 1 in $T_{e}$.

Next, we repeat the process at level 1 . Provided $\varphi_{e}(1) \downarrow$, we assign to each individual node at level 1 in $T_{e}$ one of the nodes at level 1 in $S_{e}$ and repeat this process with that node in place of the root. We then repeat this process (unless it terminates) at each level. Of course, if $\varphi_{e}$ computes a tree $S_{e}$ which is not finite-branching, then our $T_{e}$ will be a finite tree. (Also, if $\varphi_{e}$ is not total or fails to compute a tree, then again our $T_{e}$ will be a finite tree.) However, if $S_{e}$ is a finite-branching tree with domain $\omega$, then $T_{e}$ not only will also be a finite-branching tree, but will in fact be isomorphic to $S_{e}$.

Finally, notice that if $S_{e}$ is a tree with domain $\{0, \ldots, n\}$, then this process will build $T_{e}$ isomorphic to $S_{e}$, stopping when it finds that $\varphi_{e}(n+1) \uparrow$. Therefore, every finite tree appears on our list. So the set $\left\{T_{e}\right\}_{e \in \omega}$, given by a $\mathbf{0}^{\prime}$-computable list of indices for $\mathbf{0}^{\prime}$-computable trees, includes a presentation of every computable finite-branching tree, yet includes only trees which are isomorphic to computable finite-branching trees.

The remaining assumptions of Theorem 2.3 are readily seen to hold. The restriction $T_{i}\left\lceil s\right.$ of any $T_{i}$ in the enumeration is actually already downward closed and is an element of $\mathcal{T}$. Given $s$, let $h$ be the maximum of the heights of all trees $T_{k}\lceil s$ with $k<s$. Given $i$ and $s$, find some $j$ and $t$ such that $T_{j}\left\lceil t\right.$ contains a node at height $(h+1)$ and $T_{i}\left\lceil s\right.$ embeds into $T_{j} \uparrow t$. This $j$ and $t$ establish Assumption (1b) of Theorem 2.3. Assumption (2a) is already known to hold of finite-branching trees (see e.g. [19]). In Assumption (2b), the trees $T_{i} \uparrow t$ and $T_{j}\lceil s$ are both finite (and the size of the domain of each is $\mathbf{0}^{\prime}$-computable). So it is simple to check using $\mathbf{0}^{\prime}$ whether they are isomorphic 
and whether the first embeds into the second. Therefore, Theorem 2.3 yields a $\mathbf{0}^{\prime}$-computable classification of the family $\mathcal{T}$ by $\mathbf{0}^{\prime}$-computable indices.

\section{Computable Equivalence Structures}

An equivalence structure is simply an equivalence relation $E$ on a given domain $D$. For an equivalence structure to be computable, we require $D$ to be an initial segment of $\omega$, and $E$ a computable subset of $\omega \times \omega$. Notice that this definition specifically allows finite equivalence structures. We normally write $[x]_{E}$ for the $E$-equivalence class containing the element $x$ of the domain.

The principal distinction among equivalence structures arises from the number of infinite equivalence classes defined by the relation $E$. We denote the family of those computable equivalence structures containing exactly $n$ infinite equivalence classes by $\mathcal{E}_{n}$ (for each $n \in \omega$ ). The finite equivalence structures (with domain an initial segment of $\omega$ ) are all included in $\mathcal{E}_{0}$, and we specifically include the empty structure in $\mathcal{E}_{0}$.

5.1 Classifications by computable indices. Recall Theorem 1.3 of Goncharov and Knight, which states that there exists a computable classification of the family $\mathcal{E}_{\infty}$ of computable equivalence structures with infinitely many infinite equivalence classes. Our approach is to study the possibility of classifying $\mathcal{E}_{0}$. A classification of $\mathcal{E}_{0}$ would yield a classification of $\mathcal{E}_{n}$, for each $n<\omega$, because each structure in $\mathcal{E}_{n}$ is the disjoint union (in a unique way) of a structure containing $n$ infinite classes (and nothing else) with a structure containing no infinite equivalence classes. Putting this together with Theorem 1.3 would yield a classification of the family $\mathcal{E}$ of all computable equivalence structures.

Lemma 5.1 The isomorphism problem for the family $\mathcal{E}_{0}$ of computable equivalence structures with no infinite classes is $\Pi_{3}^{0}$-complete.

Proof. To see that the isomorphism problem is $\Pi_{3}^{0}$, notice that the equivalence relations $E_{i}$ and $E_{j}$ on $\omega$ computed by $\varphi_{i}$ and $\varphi_{j}$ are isomorphic if and only if $i$ and $j$ lie in the index set for $\mathcal{E}_{0}$ (which is readily seen to be $\Pi_{3}^{0}$ ) and, for every $n$ and $k$, each one has at least $n$ classes of size exactly $k$ if and only if the other does. For a given element to lie in a class of size exactly $k$ is $\mathbf{0}^{\prime}$-decidable, since there are no infinite classes. So the given condition is that, for every $k$ and all pairwise- $E_{i}$-inequivalent $x_{1}, \ldots, x_{n}$, there exist pairwise- $E_{j}$-inequivalent $y_{1}, \ldots, y_{n}$ such that

$$
\text { (every } \left.\left[x_{m}\right]_{E_{i}} \text { has size } k\right) \Longrightarrow\left(\text { every }\left[y_{m}\right]_{E_{j}} \text { has size } k\right) \text {, }
$$

along with the same statement with $i$ and $j$ reversed. This is $\Pi_{3}^{0}$.

For each input $e$, we build a pair of computable equivalence structures $E_{e}$ and $F_{e}$, uniformly in $e$. Neither $E_{e}$ nor $F_{e}$ will have any infinite equivalence classes, and $E_{e}$ and $F_{e}$ will be isomorphic iff $e \notin$ Cof. This will prove the lemma.

At stage 0 , each of $E_{e}$ and $F_{e}$ has one class of each finite size. At stage $s+1$, if $W_{e, s+1}=W_{e, s}$, we change nothing. If some (single) element $x$ has entered $W_{e}$ at stage $s+1$, fix the $n \geq 0$ such that the complement $\bar{W}_{e, s}$ contained exactly $n$ elements $<x$. By induction, $E_{e}$ and $F_{e}$ each contain exactly one class of size $2 n+1$, and contain the same number of classes of size $2 n+2$. We add $2 n+2$ new elements to each structure. In $E_{e}$, these new elements form a 
new class of size $2 n+2$. In $F_{e}$, one of these elements is added to the unique class of size $2 n+1$, forming a new class of size $2 n+2$, and the remaining new elements form a new class of size $2 n+1$. This is the entire construction.

Now if $e \in \mathbf{C o f}$, then for some (minimal) $n$, there are infinitely many stages $s+1$ at which the $(n+1)$-th smallest element of $\bar{W}_{e, s}$ enters $W_{e, s+1}$. Consequently, $F_{e}$ has no class of size $2 n+1$, since every $F_{e}$-class with $2 n+1$ elements eventually receives another element. However, the original $E_{e}$-class of size $2 n+1$ never receives any more elements, and so $E_{e} \nRightarrow F_{e}$. Conversely, if $e \notin \mathbf{C o f}$, then for every $n$ there is a stage $s$ such that none of the $(n+1)$ smallest elements of $\bar{W}_{e, s}$ ever enters $W_{e}$, and so the $F_{e}$-class with $2 n+1$ elements as of stage $s$ never acquires any more elements. Thus $F_{e}$ has exactly one class with $2 n+1$ elements, as does $E_{e}$. Moreover, as of stage $s$, they have the same number of classes of size $2 n+2$, and those classes never change at any subsequent stage. This holds for every $n$, so $E_{e} \cong F_{e}$ in this case, proving the lemma.

In this proof we remarked that the index set for $\mathcal{E}_{0}$ is $\Pi_{3}^{0}$. In fact, it is complete at this level.

Lemma 5.2 The index set for the family $\mathcal{E}_{0}$ of computable equivalence structures with no infinite classes is $\Pi_{3}^{0}$-complete.

Proof. Fix any $e \in \omega$. As with Lemma 5.1, we consider the "markers" on the complement of $W_{e}$ at each stage as we build the equivalence relation $E_{e}$. At each stage $s$, we add one new element $x_{s}$ to $E_{e}$, in a new $E_{e}$-class. Also, if the $n$-th marker moved at stage $s$ (and $n$ is minimal with this property), then we add another new element to $E_{e}$, in the $E_{e}$-class of $x_{n}$. (This assumes that $W_{e}$ is enumerated so that, at stage $s$, no $x \geq s$ enters $W_{e}$; thus $x_{n}$ must be defined at this stage.) This is the entire construction.

Now if $e \in \mathbf{C o f}$, fix the least $n$ such that the $n$-th marker moves at infinitely many stages. Then $x_{n}$ lies in an infinite $E_{e}$-class, and so the index of $E_{e}$ is not in the index set for computable equivalence relations with no infinite equivalence classes. On the other hand, if $e \notin \mathbf{C o f}$, then the index of $E_{e}$ does lie in this index set, since for every $n$ there is a stage after which the $n$-th marker never moves again, and so the equivalence class of each $x_{n}$ is finite for every $n$. Thus we have an $m$-reduction from Cof to the complement of the index set.

Corollary 5.3 There exists a $\mathbf{0}^{\prime \prime \prime}$-computable classification of the computable equivalence structures with no infinite classes, by computable indices.

Proof. With a $\mathbf{0}^{\prime \prime \prime}$-oracle, for each $n$, we may compute the least number $f(n)$ such that:

- $f(n)>f(n-1)$ or $n=0$; and

- $f(n)$ lies in the index set from Lemma 5.2; and

- for all $m<n,\langle f(m), f(n)\rangle$ does not lie in the isomorphism problem from Lemma 5.1.

Not only is this $f$ the required classification, but it is also strictly increasing, so its image is $\mathbf{0}^{\prime \prime \prime}$-computable.

Corollary 5.4 There exists a $\mathbf{0}^{\prime \prime \prime}$-computable classification of all computable equivalence structures by computable indices. 
Proof. Let $g_{0}$ be the computable function from Theorem 1.3, classifying all computable equivalence relations with infinitely many infinite classes. Let $f$ be the classification given in Corollary 5.3, and, for each $n>0$, define $g_{n}(x)$ to be the index of a computable equivalence structure which, on the even numbers, builds an isomorphic copy of the equivalence structure given by the index $f(x)$, and partitions the odd numbers into exactly $(n-1)$ equivalence classes, all infinite. (For the special case $n=1, g_{1}(x)$ uses all of $\omega$, not just the even numbers, to build the equivalence relation given by $f(x)$.) Finally, we define $g(\langle n, x\rangle)=g_{n}(x)$, giving a $\mathbf{0}^{\prime \prime \prime}$-computable classification $g$ of all computable equivalence structures by computable indices.

In their research leading to this article, the authors proved that for each finite nonempty subset $S \subseteq \omega$, there is no computable enumeration of the family $\mathcal{E}_{S}=\cup_{n \in S} \mathcal{E}_{n}$. However, instead of that proof, we present the proof of a stronger result, recently established by Harrison-Trainor, Melnikov, Montalbán, and one of us.

Theorem 5.5 (Harrison-Trainor, Melnikov, Miller, Montalbán) For every nonempty subset $S \subseteq \omega$, there is no computable enumeration of the family $\mathcal{E}_{S}=\cup_{n \in S} \mathcal{E}_{n}$ of all computable equivalence structures $E_{i}$ in which the number of infinite equivalence classes is an element of $S$. Hence, there is no computable classification of any such $\mathcal{E}_{S}$.

The notation here leads to some possibility of confusion. The family $\mathcal{E}_{\omega}$, with $S=\omega$, is defined here as the family of those computable equivalence structures with only finitely many infinite classes; this family should not be confused with $\mathcal{E}_{\infty}$, which is precisely its complement in $\mathcal{E}$. Every $\mathcal{E}_{S}$ in this theorem is disjoint from $\mathcal{E}_{\infty}$.

Proof. Suppose that $E_{0}, E_{1}, E_{2}, \ldots$ is a computable enumeration of some $\mathcal{E}_{S}$. We will produce a computable equivalence structure $E \in \mathcal{E}_{S}$ that is not isomorphic to any of these $E_{e}$, thereby proving the theorem. The construction of our $E$ from the given enumeration is uniform, except we fix one number $a \in S$. Below we will build an $E$ in $\mathcal{E}_{0}$ which has arbitrarily large finite classes, but also satisfies the following requirements $\mathcal{R}_{e}$ for every $e \in \omega$ :

$\mathcal{R}_{e}: \quad$ If $E_{e}$ has arbitrarily large finite classes, then there exists some $k \in \omega$ such that $E_{e}$ has a class of size $k$ and $E$ does not.

Now if $0 \notin S$, then our $E$ does not satisfy our purpose. However, one then builds an $E^{*}$ which has all the same finite classes as $E$, but also has $a$-many infinite classes. This $E^{*}$ then lies in $\mathcal{E}_{a}$, hence in $\mathcal{E}_{S}$, and the requirements $\mathcal{R}_{e}$ will then show that the given enumeration failed to list any isomorphic copy of $E^{*}$, thus proving the theorem.

Our strategy is to start listing the elements of the classes in each $E_{e}$. We begin our basic module against $E_{e}$ when we find the first element $x_{e, 1}$ in any of its equivalence classes. It will next require attention if we reach a stage at which the $\left[x_{e, 1}\right]_{E_{e}}$ has at least two elements and a new element $x_{e, 2}$ has appeared with $\left\langle x_{e, 2}, x_{e, 1}\right\rangle \notin E_{e}$. After that, it requires attention at the next stage (if any) at which $\left[x_{e, 1}\right]_{E_{e}}$ has at least three elements, $\left[x_{e, 2}\right]_{E_{e}}$ has at least two, and a new $x_{e, 3}$ has appeared that lies in neither of these classes. We 
continue in the same fashion forever, claiming that $\mathcal{R}_{e}$ will require attention at only finitely many stages. Indeed, by hypothesis, only finitely many of these $E_{e}$-classes are infinite, so eventually some $x_{e, i}$ will be found that lies in a finite class (or else $E_{e}$ consists of finitely many infinite classes and nothing else, in which case, for some $i \in \omega$, no $x_{e, i}$ ever appears). After that, $\mathcal{R}_{e}$ will require attention at most once each time the class $\left[x_{e, i}\right]_{E_{e}}$ expands, hence only finitely many more times in total.

Each time $\mathcal{R}_{e}$ requires attention, $E_{e}$ has finitely many equivalence classes $\left[x_{e, 1}\right]_{E_{e}}, \ldots,\left[x_{e, n_{e}}\right]_{E_{e}}$ so far, each with finitely many observed elements. We write $k_{e, j, s}$ for the size of $\left[x_{e, j}\right]_{E_{e}} \cap\{0,1, \ldots, s\}$ at stage $s$. The construction then ensures, until the next stage (if any) at which $\mathcal{R}_{e}$ requires attention, that $E$ contains no $E$-class of any of the sizes $k_{e, 0, s}, \ldots, k_{e, n_{e}, s}$. If $\mathcal{R}_{e}$ never again requires attention and $E_{e}$ has arbitrarily large finite classes, then one of the classes $\left[x_{e, i}\right]_{E_{e}}$ never again expands, hence contains exactly $k_{e, i, s}$ elements. In this case $E_{e}$ cannot be isomorphic to $E$, since $E$ will have no class of any of the sizes $k_{e, j, s}$ with $j \leq n_{e}$.

We combine the basic modules by a finite-injury process. Every $\mathcal{R}_{d}$ with $d>e$ will be injured at each stage at which $\mathcal{R}_{e}$ receives attention. After that stage, instead of just waiting for one element $x_{d, 1+n_{d}}$ in a new equivalence class to appear, $\mathcal{R}_{d}$ will watch for an $E_{d^{-}}$class $\left[x_{d, 1+n_{d}}\right]_{E_{d}}$ to appear which has at least $2+m_{d}$ elements in it, where $m_{d}=\max \left\{k_{e, j}: e<d, j \leq n_{e}\right\}$. Meanwhile, $E$ will create a class $\left[y_{d}\right]_{E}$ which has exactly $1+m_{d}$ elements. This class will help show that $E$ contains arbitrarily large finite classes. However, no class in $E$ will wind up with infinitely many elements (because of the finite-injury nature of the argument), and if $E_{d}$ also has arbitrarily large finite classes, then after the greatest stage at which it is injured, it will eventually produce an $x_{d, 0}$ and the diagonalization against $E_{d}$ will begin.

At stage 0 , we set every $n_{e, 0}=0$ and every $m_{e, 0}=2$. We set $y_{e}=2 e$ in the structure $E$ and make them all $E$-inequivalent to each other. We leave all other values undefined at this stage. At the start of stage $s+1$, we have numbers $n_{e, s}$ defined at the preceding stage, and for each $e$ with $n_{e, s}>0$ we have elements $x_{e, 1, s}, \ldots, x_{e, n_{e, s}, s}$ in $E_{e}$ and numbers $k_{e, 1, s}, \ldots, k_{e, n_{e, s}, s}$. We define the threshold values: $m_{0, s+1}=2$ and, for each $e \leq s$,

$$
m_{e, s+1}=\max \left(m_{e, s}, 2+\max \left\{k_{d, j, s}: d<e \& j \leq n_{d, s}\right\}\right)
$$

with $m_{e, s+1}=2$ for all $e>s$. For each $e \leq s$, we add $\left(m_{e, s+1}-m_{e, s}\right)$ new elements to $\left[y_{e}\right]_{E}$. (The definition of $m_{e, s+1}$ ensures that $m_{e, s+1} \geq m_{e, s}$, and so this step shows that $\left[y_{e}\right]_{E}$ has size exactly $\left(m_{e, s+1}-1\right)$ at this stage.)

Now we search for the least $e \leq s$ satisfying the following conditions:

- there exists some $x \leq s$ such that $[x]_{E_{e}} \cap\{0,1, \ldots, s\}$ contains at least $m_{e, s}$ elements and, for all $j=1, \ldots, n_{e, s}$, we have $\left\langle x, x_{e, j, s}\right\rangle \notin E_{e}$; and

- for every $j=1, \ldots, n_{e, s},\left[x_{e, j, s}\right]_{E_{e}} \cap\{0,1, \ldots, s\}$ contains at least $k_{e, j, s}+1$ elements. (These conditions on $j$ are vacuous if $n_{e, s}=0$.)

If no such $e \in \omega$ is found, then we do nothing. Otherwise, for the least such $e$, requirement $\mathcal{R}_{e}$ receives attention, as follows. We define $n_{e, s+1}=1+n_{e, s}$ (writing $n=n_{e, s+1}$ hereafter) and set $x_{e, n, s+1}$ to be the least $x$ witnessing 
the first condition above. For each $j \leq n$, we let $x_{e, j, s+1}=x_{e, j, s}$ and reset

$$
k_{e, j, s+1}=\left|\left[x_{e, j, s+1}\right]_{E_{e}} \cap\{0, \ldots, s\}\right|,
$$

noting that (by induction and the choice of $x_{e, n, s+1}$ ) every $k_{e, j, s+1} \geq m_{e, s+1}$. For every $d>e$, we reset $n_{d, s+1}=0$, thus injuring $\mathcal{R}_{d}$. (The threshold value $m_{d, s+1}$ was defined above and is preserved, but all other values associated to $\mathcal{R}_{d}$ become undefined at stage $s+1$.) The rest of $E$ is unchanged, and we preserve all values defined for all $\mathcal{R}_{d}$ with $d<e$. This completes stage $s+1$.

The argument that each $\mathcal{R}_{e}$ receives attention at only finitely many stages proceeds by induction on $e$. Fixing the least stage $s_{0}$ such that no requirement $\mathcal{R}_{d}$ with $d<e$ receives attention at any stage $t \geq s_{0}$, we note that $m_{e, s_{0}}=m_{e, s}$ for all $s>s_{0}$, and we write $m$ for this permanent threshold value for $\mathcal{R}_{e}$. Now if $E_{e}$ has no equivalence classes of size at least $m$, then $\mathcal{R}_{e}$ will never receive attention again. If $E_{e}$ does have such a class, then $x_{e, 1, s+1}$ will be defined at the first stage $s$ at which we observe such a class. Thereafter we keep on searching for more such classes and for increases in the sizes of the existing such classes. Notice that, if $x_{e, j, s}$ becomes defined after stage $s_{0}$ for some $j \in \omega$, then $x_{e, j, s}$ is never redefined, so we call it $x_{e, j}$. However, if $\mathcal{R}_{e}$ receives attention at infinitely many stages after $s_{0}$, then we would have infinitely many elements $x_{e, 1}, x_{e, 2}, \ldots$, since a new one is chosen at each such stage. Also, since each existing equivalence class $\left[x_{e, j}\right]_{E_{e}}$ must expand in order for $\mathcal{R}_{e}$ to receive attention again, every class $\left[x_{e, j}\right]_{E_{e}}$ would be infinite. Therefore, since $E_{e}$ has only finitely many infinite classes, there must exist a greatest stage $s_{1}$ at which $\mathcal{R}_{e}$ receives attention. This completes the induction.

Hence, for each $e$, the limit $m_{e}=\lim _{s} m_{e, s}$ exists. By construction, $\mathcal{R}_{e}$ stops receiving attention because either $E_{e}$ does not have any finite $E_{e}$-classes of size at least $m_{e}$ (hence $\mathcal{R}_{e}$ stopped receiving attention once representatives of all its infinite classes had been discovered), or else some $x_{e, i}$ was chosen (after the final injury to $\mathcal{R}_{e}$ ) whose equivalence class only expanded at finitely many subsequent stages (hence $\left[x_{e, i}\right]_{E_{e}}$ is finite). In the latter case, we (noneffectively) fix the index $i$ of the $x_{e, i}$ that is the first to have its $E_{e}$-class reach full size. (That is, choose $i \leq n_{e}$ so that $\max \left[x_{e, i}\right]_{E_{e}}$ is as small as possible.) Therefore, by definition of $k_{e, i, s}$, the class $\left[x_{e, i}\right]_{E_{e}}$ has size $k_{e, i}=\lim _{s} k_{e, i, s} \geq m_{e}$, and by induction on $e$ and $s$ we know that $m_{e}>m_{d}$ for all $d<e$. On the other hand, by our choice of $i, \mathcal{R}_{e}$ last receives attention at some stage $s>\max \left[x_{e, i}\right]_{E_{e}}$, and so, for every $d>e, m_{d} \geq m_{d, s+1} \geq 2+k_{e, i}$. We now show that the $E$-classes have size exactly $\left(m_{d}-1\right)$ for $d \in \omega$. Thus $E$ contains no class having the same size as $\left[x_{e, i}\right]_{E_{e}}$, and $\mathcal{R}_{e}$ is satisfied.

Every $E$-class has the form $\left[y_{d}\right]_{E}$, and once chosen, $y_{d}$ is never redefined in the construction. Moreover, as remarked in the construction, at stage $s+1, y_{d}$ lies in an $E$-class of size exactly $\left(m_{d, s+1}-1\right)$. Since each sequence $\left\langle m_{d, s}\right\rangle_{s \in \omega}$ converges to a finite value $m_{d}$ and no other $E$-classes are ever created, it is clear that the values $m_{d}-1$ are exactly the final sizes of the equivalence classes in $E$. Thus $E$ has only finite equivalence classes, and every $\mathcal{R}_{e}$ holds, as claimed above. Finally, this result also shows that there are arbitrarily large finite $E$-classes, since $m_{d}<m_{d+1}$ holds for all $d \in \omega$. So, even if $E_{e}$ satisfies $\mathcal{R}_{e}$ by virtue of having an upper bound on the size of the finite $E_{e}$-classes, we still see that $E_{e}$ and $E$ cannot be isomorphic. 
When one allows the elements of $\mathcal{E}_{\infty}$ into the enumeration as well, things become more feasible. In [10, Corollary 5.2], Goncharov and Knight gave a computable enumeration of $\mathcal{E}$, the family of all computable equivalence structures (including those with finite domains), simply by enumerating all c.e. subsets of a computable equivalence structure with infinitely many infinite classes and no finite classes. There may still exist a computable classification of $\mathcal{E}$. By Theorem 5.5, if such a classification exists, one would not be able to partition it effectively into the subfamilies $\mathcal{E}_{\infty}$ (already effectively classified by Goncharov and Knight) and $\mathcal{E}_{\omega}$. Since the isomorphism problem for $\mathcal{E}$ is $\Pi_{4}^{0}$-complete (see [3, Theorem 3.13]), Proposition 1.5 (or even the strong version given in Corollary 3.3) does not apply to $\mathcal{E}$, and so the question of computable classifiability of $\mathcal{E}$ does not yield to any of the methods used in this article. We regard this question as challenging.

5.2 Oracle classifications. In this section, we show that there is a $\mathbf{0}^{\prime}$-computable classification, by $\mathbf{0}^{\prime}$-computable indices, of the family $\mathcal{E}$ of all computable equivalence structures and other related subfamilies. First, we construct such a classification for $\mathcal{E}_{0}$, the family of all computable equivalence structures with no infinite classes. To accomplish this, we will set $\boldsymbol{d}$ to equal the degree $\mathbf{0}^{\prime}$ and apply Theorem 2.3. Specifically, we will build a $\mathbf{0}^{\prime}$-computable enumeration $\left\langle F_{e}\right\rangle_{e \in \omega}$ of $\mathcal{E}_{0}$, in such a way that the isomorphism problem $\left\{\langle i, j\rangle: F_{i} \cong F_{j}\right\}$ is $\Pi_{3}^{0}$, and thus $\Pi_{2}^{0}$ relative to our oracle, and so that the other hypotheses of Theorem 2.3 are also satisfied.

To build $F_{e}$, we consider the partial computable function $\varphi_{e}$, writing $E_{e}$ for the binary relation $\left\{\langle x, y\rangle: \varphi_{e}(\langle x, y\rangle) \downarrow=1\right\}$. With a $\mathbf{0}^{\prime}$-oracle, we may enumerate any witnesses which show that $\varphi_{e}$ fails to compute a (total) equivalence relation on $\omega$ : either a pair $\langle x, y\rangle$ for which $\varphi_{e}(\langle x, y\rangle) \uparrow$, or pairs that witness the failure of reflexivity, symmetry, or transitivity.

To start computing $F_{e}$ below $\mathbf{0}^{\prime}$, we first set $x_{0}=0, z_{0}=0$, and $n_{0}=1$, and ask our oracle whether there exist two distinct elements of $\omega$ (including $x_{0}$ itself) that are both $E_{e}$-equivalent to $x_{0}$. If not, then $z_{0}=0$ enters $\operatorname{dom}\left(F_{e}\right)$ and forms a singleton class in $F_{e}$. If so, then we increment $n_{0}$ to 2 at stage $s=1$ and ask whether there exist three distinct such elements. This process continues until either

- we find a witness showing that $E_{e}$ is not a total equivalence relation on $\omega$, in which case the construction ends here, and $F_{e}$ is a finite equivalence relation consisting of the classes already built; or

- we find the least number $n_{0}$ for which the $E_{e}$-class of $x_{0}$ fails to contain $\left(n_{0}+1\right)$ distinct elements. If this happens at stage $s$, then we set $z_{1}=z_{0}+n_{0}$ adjoin the numbers $z_{0}, \ldots, z_{0}+n_{0}-1$ to $\operatorname{dom}\left(F_{e}\right)$, and make them all $F_{e}$-equivalent to $z_{0}$, so that $z_{0}$ now lies in an $F_{e}$-class of size $n_{0}$ (just as $x_{0}$ does in $E_{e}$ ). This $F_{e}$-class will never grow any further. (Notice that $z_{1}$ is not yet in $\operatorname{dom}\left(F_{e}\right)$.)

If the second possibility holds, we now continue by finding using $\mathbf{0}^{\prime}$ the least $x_{1}>x_{0}$ such that $\left(\forall y<x_{1}\right)\left\langle x_{1}, y\right\rangle \notin E_{e}$. We run the same process with $x_{1}$, potentially finding a number $n_{1}$ at some stage $s$ as in the second possibility, in which case we set $z_{2}=z_{1}+n_{1}$, and make $z_{1}$ part of an $F_{e}$-class $\left\{z_{1}, \ldots, z_{2}-1\right\}$ of size $n_{1}$. We continue in this manner through all $x_{t}$ and $z_{t}$. 
Of course, the process above (for a particular $x_{t}$ and $z_{t}$ ) could run for infinitely many stages $s$, if $E_{e}$ is a total equivalence relation in which $x_{t}$ is the least element belonging to an infinite $E_{e}$-class. If this happens, then $F_{e}$ is exactly the equivalence relation defined by the process, comprising the finitely many finite classes built before we reached $x_{t}$. The same happens if $E_{e}$ turns out not to be a total equivalence relation. (Indeed, $F_{e}$ could turn out to be the empty equivalence structure, with domain $\emptyset$, for instance if 0 lies in an infinite $E_{e}$-class. This is why the empty structure is included in $\mathcal{E}_{0}$.) On the other hand, if $E_{e}$ is a total equivalence relation with no infinite classes, then this process builds $F_{e} \cong E_{e}$. In all cases, $F_{e}$ is an equivalence relation on an initial segment of $\omega$ and is $\mathbf{0}^{\prime}$-computable uniformly in $e$. Thus we have a $\mathbf{0}^{\prime}$-computable enumeration of $\mathbf{0}^{\prime}$-indices of $\mathcal{E}_{0}$ (which includes all finite equivalence relations, even the empty relation.) The domain of each $F_{e}$ is $\mathbf{0}^{\prime}$-computably enumerable uniformly in $e$, but its size is not. The family of all these domains, while uniformly c.e. in $\mathbf{0}^{\prime}$, is not uniformly $\mathbf{0}^{\prime}$-computable.

Recall Lemmas 5.1 and 5.2, which showed that the isomorphism problem and the index set for $\mathcal{E}_{0}$ are both $\Pi_{3}^{0}$-complete. Since the structures $F_{e}$ are only $\mathbf{0}^{\prime}$-computable, we would expect the isomorphism problem $F_{i} \cong F_{j}$ to be $\Pi_{3}^{0}$ relative to $\mathbf{0}^{\prime}$, which is to say, $\Pi_{4}^{0}$. However, the construction of the structures $F_{e}$ has an additional feature: for each $x \in \operatorname{dom}\left(F_{e}\right)$, the size of the $F_{e}$-class of $x$ is $\mathbf{0}^{\prime}$-computable. We can exploit this fact to prove the following.

Lemma 5.6 With this construction, the set $I=\left\{\langle i, j\rangle: F_{i} \cong F_{j}\right\}$ is $\Pi_{3}^{0}$.

Proof. Fixing a $\mathbf{0}^{\prime}$ oracle, we show that $I$ is $\Pi_{2}^{0}$ relative to this oracle. Notice that, whenever $z_{t+1}$ is defined in the construction of $F_{i}$, the $\mathbf{0}^{\prime}$-oracle knows the size $n_{t}$ of $\left[z_{t}\right]_{F_{i}}$, since no more elements will ever join this class. That is, the function $t \mapsto n_{t}$ is partial $\mathbf{0}^{\prime}$-computable, with domain $\left\{t: z_{t} \in F_{e}\right\}$. We will write $y_{t}$ instead of $z_{t}$ for elements of the equivalence structure $F_{j}$, and use $m_{t}$ for the size of $\left[y_{t}\right]_{F_{j}}$. Then $F_{i} \cong F_{j}$ iff, for all finite subsets of $\omega\left\{u_{1}<\cdots<u_{k}\right\}$ and all $r>0$ such that the construction of $F_{i}$ defines $n_{u_{1}}=\cdots=n_{u_{k}}=r$, there exist $v_{1}<\cdots<v_{k}$ such that the construction of $F_{j}$ defines $m_{v_{1}}=\cdots=m_{v_{k}}=r$, and if the converse statement (with the roles of $F_{i}$ and $F_{j}$ interchanged) also holds. This is $\Pi_{2}^{0}$ in the constructions of $F_{i}$ and $F_{j}$, which are $\mathbf{0}^{\prime}$-computable uniformly in $i$ and $j$.

To apply Theorem 2.3, we will expand each structure $F_{e}$ to an augmented structure $\widetilde{F}_{e}$ in a larger language. $\widetilde{F}_{e}$ will still be $\mathbf{0}^{\prime}$-computable uniformly in $e$. The expanded language has unary relation symbols $R_{1}, R_{2}, \ldots$ and unary function symbols $f_{1}, f_{2}, \ldots$, along with the binary relation $E$ from the original language. Each $\widetilde{F}_{e}$ will satisfy the following axioms:

$E$ is an equivalence relation.

$(\forall n)(\forall x)\left[R_{n}(x) \Longleftrightarrow\left([x]_{E}\right.\right.$ contains exactly $n$ elements $\left.)\right]$.

$(\forall k)(\forall x)\left[x E f_{k}(x)\right]$.

$(\forall n)(\forall j \leq n) \quad(\forall x)(\forall y)\left[\left(R_{n}(x) \& x E y\right) \Longrightarrow f_{j}(x)=f_{j}(y)\right]$.

$(\forall n)(\forall j<k \leq n) \quad(\forall x)\left[R_{n}(x) \Longrightarrow\left(f_{j}(x) \neq f_{k}(x)\right)\right]$.

$(\forall n)(\forall k>n) \quad(\forall x)\left[R_{n}(x) \Longrightarrow f_{k}(x)=x\right]$. 
These axioms imply that if $R_{n}(x)$ holds, then $f_{1}(x), \ldots, f_{n}(x)$ are precisely the distinct elements of $[x]_{E}$ and $f_{k}(x)=x$ for all $k>n$. When we expand $F_{e}$ to $\widetilde{F}_{e}$, our $\mathbf{0}^{\prime}$-oracle knows the (finite) size of the $E$-class of each $z \in F_{e}$, hence can decide the unique $n$ for which $R_{n}(z)$ holds and can find all the elements of $[z]_{F_{e}}$. We define the functions $f_{k}$, again with a $\mathbf{0}^{\prime}$-oracle, so that $f_{1}(z)<f_{2}(z)<\cdots<f_{n}(z)$ are the elements of $[z]_{F_{e}}$, and with $z=f_{k}(z)$ for all $k>n$, as required. This ensures that, if $F_{i} \cong F_{j}$, then there exists an isomorphism from $\widetilde{F}_{i}$ onto $\widetilde{F}_{j}$ as well. Conversely, of course, an isomorphism from $\widetilde{F}_{i}$ onto $\widetilde{F}_{j}$ must restrict to an isomorphism from $F_{i}$ onto $F_{j}$.

The point of these axioms is that now each $z \in F_{e}$ generates its own $F_{e}$-equivalence class (and nothing more). We claim that now all hypotheses of Theorem 2.3 are satisfied by the $\mathbf{0}^{\prime}$-computable enumeration $\left\langle\widetilde{F}_{e}\right\rangle_{e \in \omega}$. Recall that $\widetilde{F}_{i} \uparrow s$ denotes the substructure of $\widetilde{F}_{i}$ generated by the subset $\{0, \ldots, s-1\} \cap \operatorname{dom}\left(\widetilde{F}_{i}\right)$. Each $\widetilde{F}_{i} \uparrow s$ is an element of our enumeration (up to isomorphism, which is all that is necessary; it would be harmless to expand the enumeration to include not just every $\widetilde{F}_{i}$ but also every $\left.\widetilde{F}_{i} \uparrow s\right)$. Moreover, given any $i$ and $s$, every $\widetilde{F}_{k} \uparrow s$ with $k<s$ is a finite equivalence structure (in our expanded language), and so, to find the $j$ required by Assumption (1b) of the theorem, we simply form a new $\widetilde{F}$ by adjoining to $\widetilde{F}_{i} \uparrow s$ one new equivalence class of size larger than any class in every one of the $\widetilde{F}_{k} \uparrow s$. Defining the $R_{n}$ and $f_{n}$ on this $\widetilde{F}$ is easy, and our enumeration must include some $\widetilde{F}_{j} \cong \widetilde{F}$, so we pick this $j$ along with $t$ large enough that $\widetilde{F}_{j}\left\lceil t=\widetilde{F}_{j}\right.$. Clearly this $j$ and $t$ satisfy (1b).

Now suppose that $\widetilde{F}_{i}$ and $\widetilde{F}_{j}$ have the property that every $\widetilde{F}_{i} \uparrow s$ embeds into $\widetilde{F}_{j}$. This simply means that, for every $n \in \omega, \widetilde{F}_{j}$ has at least as many $F_{j}$-classes of size exactly $n$ as $\widetilde{F}_{i}$ has. If the same holds with $i$ and $j$ reversed, then clearly $F_{i} \cong F_{j}$, and we saw above that this implies $\widetilde{F}_{i} \cong \widetilde{F}_{j}$. Thus Assumption (2a) of Theorem 2.3 holds. Also, for any $s$ and $t$, we can determine from $\langle i, t, j, s\rangle$ (and our $\mathbf{0}^{\prime}$-oracle) the exact number of classes of each size $n$ in each of $\widetilde{F}_{i} \uparrow t$ and $\widetilde{F}_{j}\lceil s$ (as well as an upper bound on the sizes we need to consider, since for each $z \in \operatorname{dom}\left(\widetilde{F}_{i}\right) \cap\{0, \ldots, t-1\}$ we can find the unique $n$ for which $R_{n}(z)$ holds, and likewise for $\widetilde{F}_{j}$ ). From this information, it is immediate to see whether $\left.\widetilde{F}_{i}\right\rceil t$ embeds into $\widetilde{F}_{j}\lceil s$ (since this just means that the latter has at least as many classes of each single size as the former), and also whether they are isomorphic (which is equivalent to each one embedding into the other).

Now Theorem 2.3 yields a $\mathbf{0}^{\prime}$-computable classification of the (augmented) structures in the enumeration $\left\langle\widetilde{F}_{e}\right\rangle_{e \in \omega}$. This classification is easily stripped back down to simple equivalence structures once again, without introducing any new isomorphisms (since $F_{i} \cong F_{j}$ iff $\widetilde{F}_{i} \cong \widetilde{F}_{j}$ ). Thus $\mathcal{E}_{0}$ has a $\mathbf{0}^{\prime}$ computable classification, by $\mathbf{0}^{\prime}$-computable indices.

We state this result and use it to show that such classifications also exist for $\mathcal{E}$ and other related subfamilies.

Theorem 5.7 There exists a $\mathbf{0}^{\prime}$-computable classification, by $\mathbf{0}^{\prime}$-computable indices, of the family $\mathcal{E}_{0}$ of all computable equivalence structures with no infinite equivalence classes. Moreover, there also exist such classifications of the 
families $\mathcal{E}_{n}, \mathcal{E}_{\leq n}$ (for every $n \in \omega$ ), $\mathcal{E}_{\omega}$ (the family of all computable equivalence structures with only finitely many infinite classes), and $\mathcal{E}$ itself, the family of all computable equivalence structures.

Proof. For $\mathcal{E}_{0}$ this was established above. From the classification for $\mathcal{E}_{0}$, one immediately can build $\mathbf{0}^{\prime}$-computable classifications of each $\mathcal{E}_{n}$, uniformly in $n$, just by adding $n$-many infinite classes to each member of the classification of $\mathcal{E}_{0}$. By the uniformity, one gets the classifications of $\mathcal{E}_{\omega}$ and $\mathcal{E}_{\leq n}$ for every $n \in \omega$. The $\mathbf{0}^{\prime}$-computable classification of $\mathcal{E}$ itself is obtained by combining the classification of $\mathcal{E}_{\omega}$ with the computable classification of its complement $\mathcal{E}_{\infty}$ given by Theorem 1.3.

\section{References}

[1] C. Ash \& J.F. Knight; Computable structures and the hyperarithmetical hierarchy (Amsterdam: North-Holland Publishing Company, 2000).

[2] W. Calvert; Algebraic Structure and Computable Structure, PhD thesis, University of Notre Dame, 2005.

[3] W. Calvert, D. Cenzer, V. Harizanov, \& A. Morozov; Effective categoricity of equivalence structures, Ann. Pure Appl. Logic 141 (2006) 1-2, 306-325.

[4] W. Calvert, V. Harizanov, J.F. Knight, \& S. Miller; Index sets for computable structures, Algebra and Logic 45 (2006), 61-78.

[5] W. Calvert \& J.F. Knight; Classification from a computable viewpoint, Bulletin of Symbolic Logic 12 (2006) 2, 191-218.

[6] R.J. Coles, R.G. Downey \& T.A. Slaman; Every set has a least jump enumeration, Journal of the London Mathematical Society 62 (2000) 2 641-649.

[7] Yu.L. Ershov; Theorie der Numerierungen, Zeits. Math. Logik Grund. Math. 23 (1977), 289-371.

[8] R.M. Friedberg; Three theorems on recursive enumeration. I. Decomposition. II. Maximal set. III. Enumeration without duplication, Journal of Symbolic Logic 23 (1958) 3, 309-316.

[9] A. Frolov, I. Kalimullin, \& R. Miller; Spectra of algebraic fields and subfields, Mathematical Theory and Computational Practice: Fifth Conference on Computability in Europe, CiE 2009, eds. K. Ambos-Spies, B. Löwe, \& W. Merkle, Lecture Notes in Computer Science 5635 (Berlin: Springer-Verlag, 2009), 232241. (For Appendix A, see qcpages.qc. cuny.edu/ ${ }^{\text {rmiller/research.html.) }}$

[10] S.S. Goncharov \& J.F. Knight; Computable structure and non-structure theorems, Algebra and Logic 41 (2002) 6, 351-373.

[11] S.S. Goncharov, S. Lempp, \& D.R. Solomon; Friedberg numberings of families of of $n$-computably enumerable sets, Algebra and Logic 41 (2002) 2, 81-86.

[12] D.R. Hirschfeldt, B. Khoussainov, R.A. Shore, \& A.M. Slinko; Degree spectra and computable dimensions in algebraic structures, Annals of Pure and Applied Logic 115 (2002), 71-113.

[13] A. Kach, K. Lange, \& D. Solomon; Degrees of orders on torsion-free abelian groups, Annals of Pure and Applied Logic 164 (2013) 7-8, 822-836.

[14] L. Kronecker; Grundzüge einer arithmetischen Theorie der algebraischen Größen, J. f. Math. 92 (1882), 1-122.

[15] R. Miller; $\boldsymbol{d}$-Computable categoricity for algebraic fields, The Journal of Symbolic Logic 74 (2009) 4, 1325-1351.

[16] R. Miller \& A. Shlapentokh; Computable categoricity for algebraic fields with splitting algorithms, to appear in Trans. Amer. Math. Soc. 
[17] M. Rabin; Computable algebra, general theory, and theory of computable fields, Transactions of the American Mathematical Society 95 (1960), 341-360.

[18] R.I. Soare; Recursively Enumerable Sets and Degrees (New York: SpringerVerlag, 1987).

[19] R.M. Steiner; Effective algebraicity, Arch. Math. Logic 52 (2013), 91-112.

\section{Acknowledgments}

The first author was partially supported by grants numbered DMS-0802961 and DMS-1100604 from the National Science Foundation. The second author was partially supported by several grants from The City University of New York PSC-CUNY Research Award Program, and by a grant from the Queens College Research Enhancement Program. The second and third authors were partially supported by grant numbered DMS-1001306, and the second author also by DMS-1362206, from the National Science Foundation. All authors wish to acknowledge Richard Shore for useful conversations and Matthew HarrisonTrainor, Alexander Melnikov, and Antonio Montalbán for allowing us to include Theorem 5.5 here. They also thank the referee for pointing out some important corrections to Lemma 2.4.

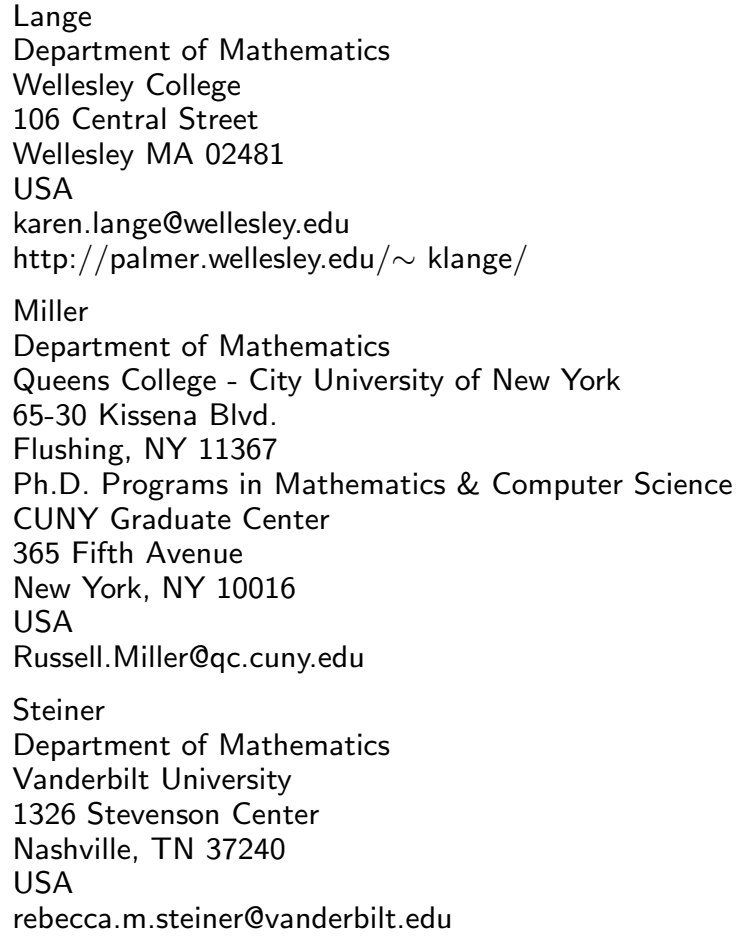

